\title{
Nonlocal plasticity based damage modeling in quasi-brittle materials using an Isogeometric approach
}

\author{
A. Rawat ${ }^{1}$, P. Raghu ${ }^{1}$, A. Rajagopal ${ }^{* 1}$, and M. Hossain ${ }^{2}$ \\ ${ }^{1}$ Indian Institute of Technology Hyderabad, India \\ ${ }^{2}$ College of Engineering, Swansea University, UK.
}

\begin{abstract}
Continuum damage mechanics allows us to define the micro damage associated with diffused fracture or damage processes in quasibrittle materials. In such strain softening materials the nonlocal and gradient enhanced approaches help to over come the effects of mesh dependednt results which are observed in local models. In this work we propose a nonlocal elastic plastic damage model. The nonlocal nature of the strain field provides a regularization to overcome the analytical and computational problems induced by softening constitutive laws. An additive decomposition of strains in to elastic and inelastic or plastic part is considered. In order to obtain stable damage, a higher gradient order is considered for an integral equation, which is obtained by the Taylor series expansion of the local inelastic strain around the point under consideration. The higher order continuity of NURBS functions used in Isogeometric analysis are adopted here to implement in a numerical scheme. To demonstrate the validity of the proposed model, numerical examples in one and two dimensions are presented.
\end{abstract}

Keywords: Nonlocal plasticity, Gradient damage model, Damage Mechanics.

\section{Introduction}

In continuum damage mechanics the development of micro-structural material damage is represented in continuum sense by a field variable ( [1] and [2]). A crack gets initiated when this local damage variable at a material point reaches a critical value and all the strength is lost at that point. The point experiences a transition from initial smooth strain to a highly localized one. The evolution depends upon the type of loading. Typically, this strain evolution occurs in a narrow band along with the coalesce of micro cracks. With progress in loading, this damage variable propagates by a process of damage growth and stress redistrbution. The rate of propagation and its direction are governed by the damage growth in a relatively small process zone in front of the crack [3]. This process of crack

*rajagopal@iith.ac.in 
modelling is termed as a local approach to modeling fracture. Numerical analysis based on local damage models result in mesh dependendent non- physical inconsistent results, that the localization of damage happens over a vanishing volume [4]. In the limit of an infinite spatial resolution, the predicted damage band has a thickness zero and the crack growth becomes instantaneous. The response is then perfectly brittle, i.e., no work is needed to complete the fracture process. These are over come by nonlocal models.

Nonlocal approaches involve a finite neighborhood volume integral of the damage variable at a material point [5]. Such an approach of spatially averaging the quantities results in smooth damage fields [6], in which the localization of damage is limited to the length scale introduced by the averaging. As a consequence, premature initiation of cracks is avoided and predicted crack growth rates remain finite. There are several notable works on nonlocal continuum theories (see works by Eringen [7] and [8], Jirasek [9], Reddy [10], and [11]). An alternative to nonlocal formulations is considering gradients of the nonlocal variable by making use of Taylor series approximations (see [12], [13], 14] and [15]). Such theories are called gradient continuum theories (for instance see [16], [17], [18] and [19]). A comparison of various higher order gradient theories can be found in [20]. Closely related to the gradient approaches are the phase field models. Phase-field method has become an efficient framework for heterogeneous systems by introducing diffuse interface concept into micro-structural simulation. This method provides an excellent tool for studying the effects of the micro-structure evolution without the necessity of tracking the sharp interface. The advantage of using this method is that it is relatively simple for implementation. In this method, damage is measured by a scalar field (phase field) [21]. Phase field method has been extensively used for studying crack propagation and fracture models (see [22], [23]). This technique has also been used to study the evolution of damage in the material (see [24]).

Models based on pure damage or pure plasticity models although yield a satisfactory result for concrete in monotonic loading conditions but fail to capture the responses under reloading and unloading conditions (see [44] and [45]). There has been a recent works with the goal of simulating the evolution of the stimulated volume during hydraulic fracturing [25] in rocks. This was achieved by introducing an equivalent continuum non-local poro-elasticplastic zone of enhanced permeability for the stimulated region, characterized by an internal length scale [26]. Hence, it is crucial to incorporate the damage while studying the softening behavior in concrete [27] and are able to more precisely model crack propagation in concrete ( see [28], 29] and [30]). There are nonlocal models which deal with either fracture energy dissipated in active cracks or with a dimension of damage in active cracks [31]. Non local elastic plastic models are able to precisely capture the damage and sotening behavior more accurately (see [32] and [33] and [34).

Isogeometric approach (IGA) [35] is employed for higher order gradient damage models. There are significant advantages from the very smooth B - spline functions, especially for solving higher order differential equations [36]. The present work includes the formulation and implementation of a nonlocal damage plasticity model using an isogeometric discretization (IGA), which is the novel contribution of this paper. An implicit gradient enhancement is considered to make the approximation simpler. During inelastic deformations, the proposed strain tensor partitioning allows the use of a distinct potential surface and distinct failure criterion for both damage and plasticity models [37]. The outline of this paper is as follows. In Section 2, we present the isotropic nonlocal damage formulation followed by 

Finite element models and the details of the Arc-length algorithm to solve the system of nonlinear equations are presented in Appendix. Few numerical examples in one- and two dimensions are presented in Section [6.

\section{Isotropic nonlocal damage formulation}

Let us consider a body in a real number space such that $\Omega \in \mathbb{R}^{3}$ and let $\mathbf{u}$ be the infinitesimal displacement vector defined in rectangular coordinate system. The displacements satisfy Dirichlet boundary conditions, $\mathbf{u}=\mathbf{u}_{g}$ on $\Gamma_{\boldsymbol{u}}$. The Cauchy stress tensor, $\boldsymbol{\sigma}$ are used as the corresponding stress measure, and a traction $\mathbf{t}$ that acts on the Neumann boundary $\Gamma_{t}$, and $\mathbf{t}$ is equal to the projection of the $\boldsymbol{\sigma}$ on the outward pointing normal vector $\mathbf{n}$, and is given by $\boldsymbol{\sigma} \cdot \mathbf{n}=\mathbf{t}$. In isotropic continuum damage mechanics, relation between $\boldsymbol{\sigma}$ and infinitesimal strain tensor $\varepsilon^{e}$ is given by

$$
\boldsymbol{\sigma}=[1-D] \boldsymbol{E}: \boldsymbol{\varepsilon}^{e}
$$

where $D$ is the scalar damage variable, and $\boldsymbol{E}$ is the fourth order tensor of elastic moduli. The value of $D$ is varying from 0 to 1 , i.e., $0 \leq D \leq 1 . \quad D=0$ implies an undamaged material, while $D=1$ depicts a fully damaged material. This damage variable positively depends on the history parameter $\kappa$, which is positively related to the nonlocal strain $\bar{e}$, so as the $\bar{e}$ is increasing, we can conclude that $D$ would also be increasing. As $D$ is a function of $\kappa, D(\kappa)$ would be referred to as the damage law. However, in local formulations, $D$ depends on the local strain, which provides an unstable result due to ill-conditioning of equations, see [38], [39], and [40]. According to the Kuhn-Tucker conditions for a nonlocal measure,

$$
\dot{\kappa} \geq 0, \quad \kappa-\bar{e} \leq 0, \quad \dot{\kappa}[\kappa-\bar{e}]=0
$$

The motivation behind the nonlocal theory is to obtain a stable solution since it accounts for averaging by interacting with the neighbor elements at the micro-level. Nonlocal equivalent strain is obtained through a Taylor expansion of local strain. We can avoid the higher-order terms as they do not contribute significantly.

$$
\bar{e}=e+a_{1} \boldsymbol{\nabla}^{2} e+a_{2} \boldsymbol{\nabla}^{4} e
$$

where, $a_{1}, a_{2}, \ldots$ are the coefficients and $\nabla^{2}$ is the Laplacian operator, for more details see [41. These coefficients $a_{1}$ and $a_{2}$ are of the order of the square and quartic of the length, respectively. In this model, we are approaching Galerkin formulation to obtain the analytical solution of the nonlinear equations. As the Eq.(31) has comprised higher order partial differential terms, it requires $C^{1}$ continuous approximation. An implicit form of Eq.(3) is given by,

$$
\bar{e}-a_{1} \boldsymbol{\nabla}^{2} \bar{e}+a_{2} \boldsymbol{\nabla}^{4} \bar{e}=e .
$$

To translate the partial differential equations to the boundary value problem, we need additional information on the boundary. This requires to specify $\bar{e}$ itself or the normal derivative of $\bar{e}$, that is $\boldsymbol{\nabla} \bar{e} . \mathbf{n}=0$, where $\mathbf{n}$ is unit normal vector to the boundary. 


\section{Coupled elasto-plastic nonlocal damage model}

The coupled damage and plasticity constitutive equations are arrived by following an additive decomposition of the total strain field into elastic and plastic parts, i.e., $\varepsilon=\varepsilon^{e}+\varepsilon^{p}$. The plastic deformation shows the material has yielded while the damage depicts the crack pattern in a body, so the stored strain energy during deformation can be written as the sum of elastic strain energies and inelastic strain energy due to plastic deformation and damage deformation, and with an appropriate yield criteria one can determine the inelastic deformation. For this purpose, the onset of plastic deformation and damage would describe the entire loading process. In this model, a nonlocal plasticity model with kinematic hardening is introduced. A plastic yield function $F(\boldsymbol{\sigma}, \boldsymbol{q})$ which depends on the stress $\boldsymbol{\sigma}$ and dissipative thermodynamic force $\boldsymbol{q}$ is considered. The back stress $\boldsymbol{q}$ is associated with the internal hardening variable $\boldsymbol{\alpha}$ by the relation,

$$
\boldsymbol{q}=[1-D] \boldsymbol{H}: \boldsymbol{\alpha}
$$

where, $\boldsymbol{H}$ is the 4 th-order tensor of plastic moduli. The evolution in stress is given by

$$
\dot{\boldsymbol{\sigma}}=[1-D] \boldsymbol{E}:\left[\dot{\boldsymbol{\varepsilon}}-\dot{\varepsilon}^{p}\right]-\dot{D} \boldsymbol{E}:\left[\varepsilon-\varepsilon^{p}\right] .
$$

Similarly, the evolution of the back stress is given by

$$
\dot{\boldsymbol{q}}=[1-D] \boldsymbol{H}: \dot{\boldsymbol{\alpha}}-\dot{D} \boldsymbol{H}: \boldsymbol{\alpha}
$$

The flow and hardening rules, respectively, are given by

$$
\begin{aligned}
& \dot{\boldsymbol{\varepsilon}}^{p}=\dot{\lambda} \frac{\partial F(\boldsymbol{\sigma}, \boldsymbol{q})}{\partial \boldsymbol{\sigma}} \\
& \dot{\boldsymbol{\alpha}}=\dot{\lambda} \frac{\partial F(\boldsymbol{\sigma}, \boldsymbol{q})}{\partial \boldsymbol{q}} .
\end{aligned}
$$

where, $\lambda$ is a internal variable.

Kuhn-Tucker complementary conditions are given by

$$
\dot{\lambda} \geq 0, \quad F(\boldsymbol{\sigma}, \boldsymbol{q}) \leq 0, \quad \dot{\lambda} F(\boldsymbol{\sigma}, \boldsymbol{q})=0
$$

In order to solve Eq.(8) and Eq.(91), a standard predictor-corrector solution procedure is adopted. In the predictor phase, Eq.(8) and Eq.(19) will be solved by freezing the plastic process, while in the corrector phase, the plastic field increment is evaluated. The total time interval is discretized as $[0, T]=U_{i=1}^{N}\left[t_{i-1}, t_{i}\right]$. It is assumed that the yield function at any time $t=t_{i}$ satisfies the Kuhn-Tucker conditions and represents an admissible state of stress [42]. Following an operator split concept, a stress equilibrium Eq.(11) is decomposed into elastic and inelastic parts, leading to the corresponding numerical algorithm including an elastic-predictor and a plastic-corrector [43]. 


\subsection{Numerical integration of nonlocal plasticity model}

As stated above, in the corrector phase, the plastic part is frozen, but this assumption does not correspond to any actual admissible state of stress unless the incremental process is entirely elastic. So the resultant equation would be,

$$
\varepsilon_{i}^{\mathrm{p}, \text { trial }}=\varepsilon_{i-1}^{p}
$$

and

$$
\boldsymbol{\sigma}_{i}^{\text {trial }}=\left[1-D_{i-1}\right] \boldsymbol{E}:\left[\boldsymbol{\varepsilon}_{i}-\boldsymbol{\varepsilon}_{i-1}^{\mathrm{p} \text {,trial }}\right]=\boldsymbol{\sigma}_{i-1}+\left[1-D_{i-1}\right] \boldsymbol{E}: \Delta \boldsymbol{\varepsilon}_{i}^{p}
$$

In order to verify the corrector phase assumption, the trial stress is substituted into the yield function $F(\boldsymbol{\sigma}, \boldsymbol{q})$, and if $F(\boldsymbol{\sigma}, \boldsymbol{q}) \leq 0$, the process is elastic and there would not be any increment in plastic process. For example,

$$
\boldsymbol{\alpha}_{i}=\boldsymbol{\alpha}_{i-1}, \quad \boldsymbol{q}_{i}=\boldsymbol{q}_{i-1}, \quad \boldsymbol{\varepsilon}_{i}^{p}=\varepsilon_{i-1}^{p} .
$$

However, if $F(\boldsymbol{\sigma}, \boldsymbol{q})>0$, the trial state would violate the Kuhn-Tucker loading/unloading conditions by lying outside the yield surface. In that case, the consistency condition would be applied to rectify the trial state by return-mapping/plastic-corrector step. Thus, we require $\Delta \lambda>0$.

By using the consistency condition and return mapping algorithm $\Delta \lambda$ can be determined as

$$
\begin{gathered}
\dot{F}(\boldsymbol{\sigma}, \boldsymbol{q})=\frac{\partial F(\boldsymbol{\sigma}, \boldsymbol{q})}{\partial \boldsymbol{\sigma}}: \dot{\boldsymbol{\sigma}}+\frac{\partial F(\boldsymbol{\sigma}, \boldsymbol{q})}{\partial \boldsymbol{q}}: \dot{\boldsymbol{q}}=0 \\
\dot{\boldsymbol{\sigma}}_{i}=\left[1-D_{i-1}\right] \boldsymbol{E}:\left[\dot{\boldsymbol{\varepsilon}}_{i}-\dot{\lambda}_{i} \frac{\partial F_{i}}{\partial \boldsymbol{\sigma}_{i}}\right]-\dot{D}_{i-1} \boldsymbol{E}: \boldsymbol{\varepsilon}_{i-1}^{e} \\
\dot{\boldsymbol{q}}_{i}=\left[1-D_{i-1}\right] \boldsymbol{H}: \dot{\boldsymbol{\alpha}}_{i}-\dot{D}_{i-1} \boldsymbol{H}: \boldsymbol{\alpha}_{i-1}
\end{gathered}
$$

By substituting Eqs. (13) and (14) in Eq.(12), it will lead to

$$
\dot{\lambda}_{i}=\frac{\partial_{\boldsymbol{\sigma}} F:\left[1-D_{i-1}\right] \boldsymbol{E}: \dot{\boldsymbol{\varepsilon}}_{i}-\partial_{\boldsymbol{\sigma}} F: \dot{D}_{i-1} \boldsymbol{E}: \boldsymbol{\varepsilon}_{i-1}^{e}-\partial_{\boldsymbol{q}} F: \dot{D}_{i-1} \boldsymbol{H}: \boldsymbol{\alpha}_{i-1}}{\partial_{\boldsymbol{\sigma}} F:\left[1-D_{i-1}\right] \boldsymbol{E}: \partial_{\boldsymbol{\sigma}} F+\partial_{\boldsymbol{q}} F:\left[1-D_{i-1}\right] \boldsymbol{H}: \partial_{\boldsymbol{q}} F}
$$

Substitution of Eq.(15) into Eq.(13) leads to Eq.(16)

$$
\dot{\boldsymbol{\sigma}}_{i}=\left[1-D_{i-1}\right] \boldsymbol{E}_{\mathrm{ep}}: \dot{\boldsymbol{\varepsilon}}_{i}-\dot{D}_{i-1} \boldsymbol{H}_{\mathrm{ep}}: \boldsymbol{\varepsilon}_{i-1}^{e}-\boldsymbol{E}: \frac{\left[\partial_{\boldsymbol{q}} F: \dot{D}_{i-1} \boldsymbol{H}: \boldsymbol{\alpha}_{i-1}\right] \cdot \partial_{\boldsymbol{\sigma}} F}{\partial_{\boldsymbol{\sigma}} F: \boldsymbol{E}: \partial_{\boldsymbol{\sigma}} F+\partial_{\boldsymbol{q}} F: \boldsymbol{H}: \partial_{\boldsymbol{q}} F}
$$

From Eq.(16), an elasto-plastic moduli is given by

$$
\boldsymbol{E}_{\mathrm{ep}}=\left[\boldsymbol{E}-\frac{\left[\partial_{\boldsymbol{\sigma}} F: \boldsymbol{E}\right] \cdot \partial_{\boldsymbol{\sigma}} F}{\partial_{\boldsymbol{\sigma}} F: \boldsymbol{E}: \partial_{\boldsymbol{\sigma}} F+\partial_{\boldsymbol{q}} F: \boldsymbol{H}: \partial_{\boldsymbol{q}} F}\right]
$$

The term $\boldsymbol{H}_{\mathrm{ep}}$ is given as

$$
\boldsymbol{H}_{\mathrm{ep}}=\left[\boldsymbol{E}-\frac{\boldsymbol{E}:\left[\partial_{\boldsymbol{\sigma}} F: \boldsymbol{E}\right] \cdot \partial_{\boldsymbol{\sigma}} F}{\partial_{\boldsymbol{\sigma}} F: \boldsymbol{E}: \partial_{\boldsymbol{\sigma}} F+\partial_{\boldsymbol{q}} F: \boldsymbol{H}: \partial_{\boldsymbol{q}} F}\right]
$$


For the one-dimensional case, the above Eq.(16) reduces to the form,

$$
\dot{\sigma}_{i}=\left[1-D_{i-1}\right] E_{\mathrm{ep}} \dot{\varepsilon}_{i}-\dot{D}_{i-1} H_{\mathrm{ep}} \varepsilon_{i-1}^{e}-\dot{D}_{i-1}\left[\frac{E H}{E+H}\right] \alpha_{i-1} \partial_{\sigma} F
$$

where the elastic-plastic moduli is given by,

$$
E_{\mathrm{ep}}=E\left[1-\frac{E \partial_{\sigma} F}{E+H}\right] .
$$

and $H_{\mathrm{ep}}$ is given by,

$$
H_{\mathrm{ep}}=E\left[1+\frac{1}{E+H}\right]
$$

\section{Isogeometric approach for nonlocal plasticity}

A higher-order approximation has been used in Eq.(41). The order of approximation in a formulation can be reduced by considering an implicit formulation. Therefore, to implement this formulation in a computational framework, we require a numerical method that inherently has higher-order approximations, such as the isogeometric analysis (IGA). The governing equation is given in Eq.(22) while the weak form of the equation is given in Eq.(23)

$$
\nabla \cdot \sigma+f_{b}=0
$$

The continuous weak form of Eq.(22) is given by

$$
\int_{\Omega} \boldsymbol{\nabla} w_{\boldsymbol{u}}: \boldsymbol{\sigma} d \Omega=\int_{\Omega} w_{\boldsymbol{u}} \cdot \boldsymbol{f}_{\boldsymbol{b}} d \Omega+\int_{T_{t}} w_{\boldsymbol{u}} \cdot \boldsymbol{t} d T
$$

where $w(x)$ is a weighting function. The weak form of the nonlocal strain Eq. (44) is given by a similar way,

$$
\int_{\Omega} w_{\bar{e}}^{h} \bar{e} d \Omega \quad+\int_{\Omega} \nabla w_{\bar{e}} \cdot c \boldsymbol{\nabla} \bar{e} d \Omega \quad-\int_{\Omega} \nabla^{2} w_{\bar{e}} \cdot d \nabla^{2} \bar{e} d \Omega=\int_{\Omega} w_{\bar{e}} e d \Omega
$$

To solve the Eq.(23) and Eq.(24), a Galerkin method is approached, and to solve these equations, we approached B-spline approximation due to the presence of the higher-order element. These equations have been linearized and solved by an arc-length method. After making the above-mentioned implementation, stiffness matrix $\boldsymbol{K}$ and force vector $\boldsymbol{f}$ is given by (for more details see [41]),

$$
\begin{array}{rl}
\boldsymbol{K}_{i-1}^{\boldsymbol{u} u} & =\int_{\Omega^{e}} \boldsymbol{B}_{\boldsymbol{u}}^{T}\left[1-D_{i-1}\right] \tilde{\boldsymbol{E}}: \boldsymbol{B}_{\boldsymbol{u}} d \Omega^{e} \\
\boldsymbol{K}_{i-1}^{\boldsymbol{u} \bar{e}} & =-\int_{\Omega^{e}} \boldsymbol{B}_{\boldsymbol{u}}^{T}\left\{\frac{\partial D}{\partial \kappa}\right\}_{i-1}\left\{\frac{\partial \kappa}{\partial \bar{e}}\right\}_{i-1} \boldsymbol{N}_{\bar{e}} \tilde{\boldsymbol{E}}: \boldsymbol{\varepsilon}_{i-1} d \Omega^{e} \\
\boldsymbol{K}_{i-1}^{\bar{e} \boldsymbol{u}} & =-\int_{\Omega^{e}} \boldsymbol{N}_{\bar{e}}^{T}\left\{\frac{\partial e}{\partial \boldsymbol{\varepsilon}_{i-1}}\right\}^{T} \boldsymbol{B}_{\boldsymbol{u}} d \Omega^{e} \\
\boldsymbol{K}_{i-1}^{\bar{e} \bar{e}} & =\int_{\Omega^{e}} \boldsymbol{N}_{\bar{e}}^{T} \boldsymbol{N}_{\bar{e}} d \Omega^{e}+\int_{\Omega^{e}} \boldsymbol{B}_{\bar{e}}^{T} a_{1} \boldsymbol{B}_{\bar{e}} d \Omega^{e}-\int_{\Omega^{e}} \boldsymbol{G}_{\bar{e}}^{T} a_{2} \boldsymbol{G}_{\bar{e}} \bar{e} d \Omega^{e} \\
6 & 6 \\
\text { http://mc.manuscriptcentral.com/engcom }
\end{array}
$$




$$
\begin{aligned}
\left(\boldsymbol{f}_{\text {int }}^{\boldsymbol{u}}\right)_{i-1} & =\int_{\Omega^{e}} \boldsymbol{B}_{\boldsymbol{u}}^{T} \boldsymbol{\sigma}_{i-1} d \Omega^{e} \\
\left(\boldsymbol{f}_{\text {int }}^{\bar{e}}\right)_{i-1} & =\int_{\Omega^{e}} \boldsymbol{N}_{\bar{e}}^{T} e_{i-1} d \Omega^{e}-\int_{\Omega^{e}} \boldsymbol{N}_{\bar{e}}^{T} \boldsymbol{N}_{\bar{e}} \bar{e}_{i-1} d \Omega^{e}-\int_{\Omega^{e}} \boldsymbol{B}_{\bar{e}}^{T} a_{1} \boldsymbol{B}_{\bar{e}} \bar{e}_{i-1} d \Omega^{e}
\end{aligned}
$$

However, in case of $C^{0}$ continuity, there would be a change in $\boldsymbol{K}^{\bar{e} \bar{e}}$

$$
\boldsymbol{K}_{i-1}^{\bar{e} \bar{e}}=\int_{\Omega^{e}} \boldsymbol{N}_{\bar{e}}^{T} \boldsymbol{N}_{\bar{e}} d \Omega^{e}+\int_{\Omega^{e}} \boldsymbol{B}_{\bar{e}}^{T} a_{1} \boldsymbol{B}_{\bar{e}} d \Omega^{e}
$$

\section{B-spline Basis functions and NURBS}

In this section, we present the details of construction of B - spline basis and NURBS basis functions. Let $K$ be a vector containing a non-descending sequence in parameter space, $K \in \mathbb{P}$, which is defined as

$$
\begin{array}{lll} 
& K=\left\{\xi_{1}, \xi_{2}, \ldots, \xi_{n_{k}}\right\}, & \xi_{i} \in \mathbb{R} \\
\text { such that } & \xi_{i} \geqslant \xi_{i-1} & i=2,3, \ldots, n_{k}
\end{array}
$$

The vector $K$ and scalar $\xi_{i}$ are often termed as knot vector and knots, respectively, in computational geometry. Once we define the knot vector, the B-spline basis $N_{i}^{p}(\xi)$ of order $p>0$ are computed from constant basis using a recursive subdivision method.

$$
\begin{array}{ll}
\text { for } p=0 ; & N_{i}^{0}(\xi)=\left\{\begin{array}{ll}
1 & \text { if } \quad \xi \in\left[\xi_{i}, \xi_{i+1}\right) \\
0 & \text { otherwise }
\end{array}\right\} \\
\text { for } p>0 ; \quad N_{i}^{p}(\xi)=\left\{\begin{array}{ll}
\frac{\left(\xi-\xi_{i}\right)}{\left(\xi_{i+p}-\xi_{i}\right)} N_{i}^{p-1}(\xi)+\frac{\left(\xi_{i+p+1}-\xi\right)}{\left(\xi_{i+p+1}-\xi_{i+1}\right)} N_{i+1}^{p-1}(\xi) & \text { if } \quad \xi \in\left[\xi_{i}, \xi_{i+p+1}\right) \\
0 & \text { otherwise }
\end{array}\right\}
\end{array}
$$

The B-spline basis for an arbitrary knot vector does not contain interpolation functions. In order to obtain interpolation functions, knots are required to be repeated, which is referred to as the knot multiplicity. In this regard, we introduce an open knot vector containing the end knot with knot multiplicity equal to $p+1$. Further, B-spline basis includes other important properties such as:

- Partition of unity $\sum_{i=1}^{n_{c p}} N_{i}^{p}(\xi)=1 \quad \forall \quad \xi \in[0,1)$

- Non Negative $N_{i}^{p}(\xi) \geqslant 0 \quad \forall \quad \xi \in[0,1)$

- Kronecker delta $N_{i}^{p}\left(\xi_{j}\right)=\delta_{i j} \quad \xi_{j} \in\left[\xi_{i}, \xi_{i+p+1}\right)$

iff the knot multiplicity of $\xi_{j}$ equal to order of curve

The standard geometries, such as a circle or ellipse, are best represented using rational functions. In this regard, we generalize the B-spline to rational polynomial referred as nonuniform rational B-splines (NURBS):

$$
\mathrm{N}_{i}^{P}(\xi)=\frac{N_{i}^{p}(\xi) w_{i}}{\sum_{j=0}^{n_{c p}} N_{j}^{p}(\xi) w_{j}}
$$



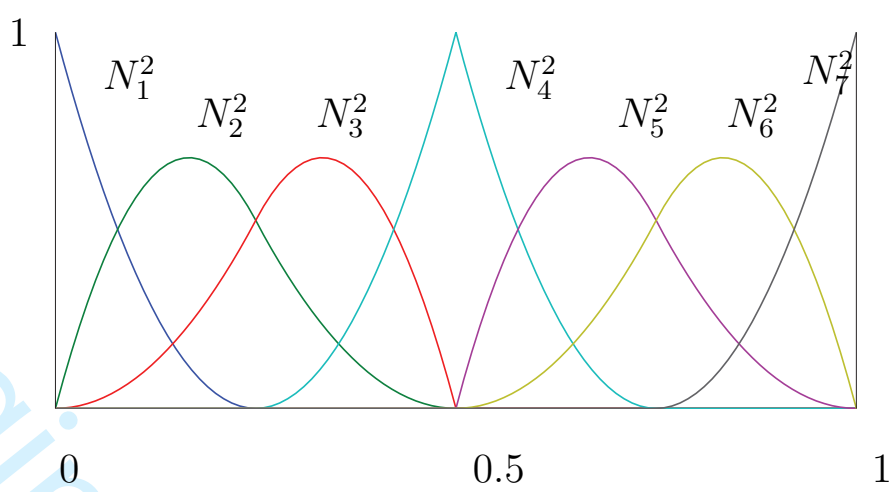

(a)

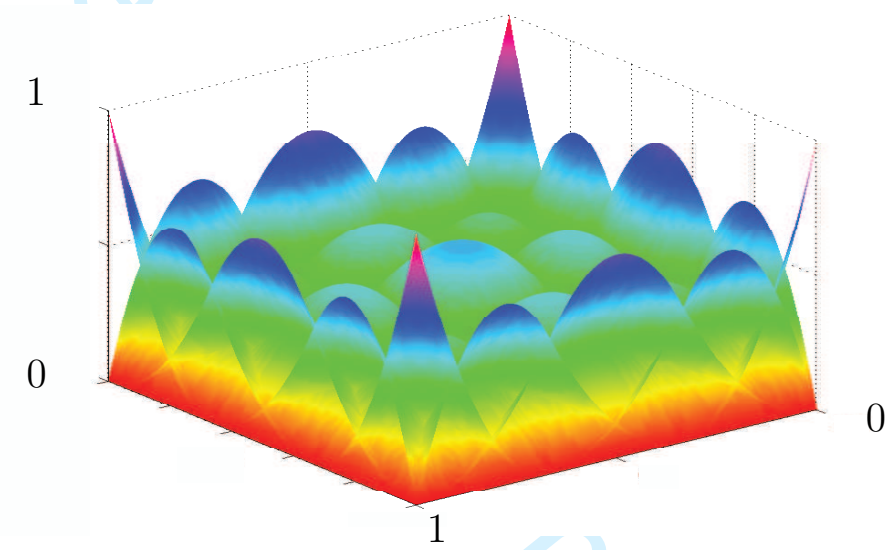

(b)

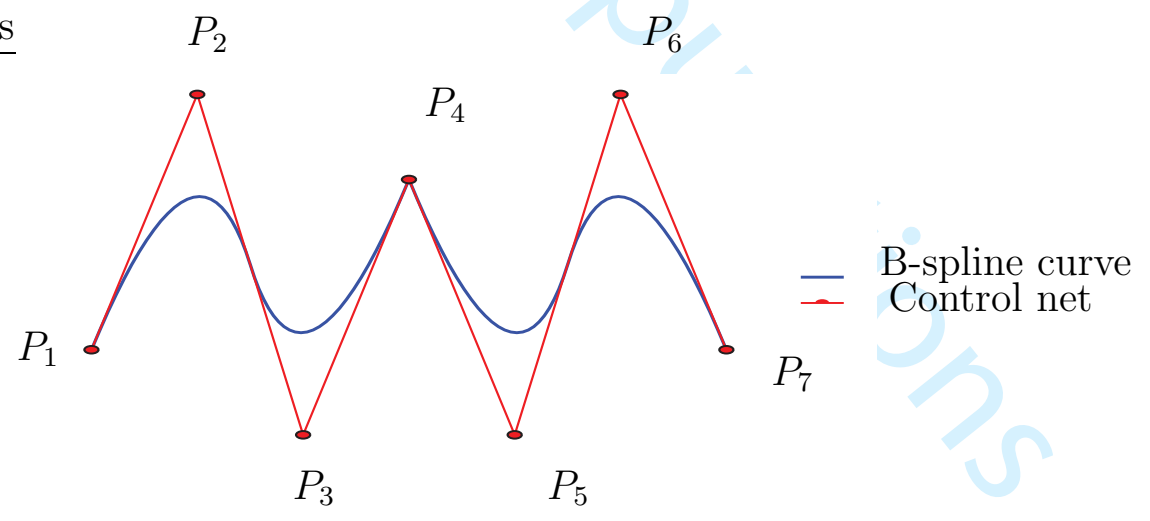

(c)

Figure 1: (a) B-spline basis functions of order 2 (b) B-spline basis functions of order 2 in two dimensions for a knot vector $K=\{0,0,0,1 / 4,2 / 4,2 / 4,3 / 4,1,1,1\}$ (c) Control net and B-spline curve 
Where $w_{i}$ are the weights associated with the control points; we note that the number of weights and control points must match. We also note that the B-spline functions are recovered from NURBS by taking all weights equal to unity. A piecewise B-spline curve is constructed by taking a linear combination of the basis vectors $N_{i}^{p}(\xi)$ and the coefficients $P_{i}$, referred to as the control points:

$$
P(x)=\sum_{i=1}^{n_{c p}} N_{i}^{p}(\xi) P_{i}
$$

The B-spline polynomials of order $p$ have $C^{p-1}$ continuous derivatives. If $k$ represents knot multiplicity at knot $\xi_{i}$, then continuous derivative of polynomial gets reduced by $C^{p-1-k}$ at that knot. Figure [ 1 shows the quadratic B-spline basis and polynomial curve for an assumed knot vector. The basis functions associated with control points $P_{1}, P_{4}$, and $P_{7}$ are interpolation functions as knots are repeated. It can also be seen in continuous derivative of B-spline polynomial. Polynomial have $C^{1}$-continuous derivative except at control points where knots are repeated making the continuity $C^{0}$, that is, at control point $P_{4}$. Using the knowledge of univariate B-spline basis helps to define the basis in multi-dimension by making use of tensor product. Let $d_{p}$ represent dimension of parametric space, $\mathbb{P}^{d_{p}}$. A knot vector defined in $\mathbb{P}^{d_{p}}$ is expressed as:

$$
K_{\mathbf{i}}=\left\{\xi_{1}^{\ell}, \xi_{2}^{\ell}, \ldots, \xi_{n_{k}^{\ell}+p^{\ell}+1}^{\ell}\right\} \quad \text { for } \quad \ell=1,2, \ldots, d_{p}
$$

where $n_{k}^{\ell}$ and $p^{\ell}$ are the knot vector dimension and basis order in $\ell^{\text {th }}$ parametric direction. Let $N_{i}^{P^{\ell}}, i=1,2$, ldots, $n_{c p}^{\ell}$, be the univariate basis function defined in $\ell^{\text {th }}$ parametric direction. Multivariate basis function are then defined through tensor product and expressed as

$$
B_{\mathbf{i}}^{\mathbf{P}}(\boldsymbol{\xi})=\prod_{\ell=1}^{d_{p}} N_{i}^{p^{\ell}}\left(\xi^{\ell}\right)
$$

where $\mathbf{i}, \boldsymbol{\xi}$ and $\mathbf{P}$ are the multi-index in parameter space. Finally, for a given control net $P_{\mathbf{i}} \in \mathbb{R}^{d}, P_{\mathbf{i}}=\left\{p_{1}^{\ell}, p_{2}^{\ell}, \ldots, p_{n_{c p}^{\ell}}^{\ell}\right\}$, the B-spline surface is defined as

$$
P(\boldsymbol{x})=\sum_{i=1}^{n_{c p}^{\ell}} B_{\mathbf{i}}^{\mathbf{P}}(\boldsymbol{\xi}) P_{\mathbf{i}}
$$

\subsection{Numerical Implementation to solve the equilibrium equations}

Following the Galerkin approach, the displacement vector $\boldsymbol{u}$ and the weight function $w$ are discretized by the interpolation matrix $\boldsymbol{N}$ containing the shape functions; the columns $\boldsymbol{u}$ and $w_{\boldsymbol{u}}$ contain the nodal displacement and weight vector components, respectively

$$
\boldsymbol{u}=\boldsymbol{N}_{\boldsymbol{u}} \boldsymbol{u} \quad \text { and } \quad \boldsymbol{\nabla} \boldsymbol{u}=\boldsymbol{B}_{\boldsymbol{u}} \boldsymbol{u}
$$

Therefore,

$$
\begin{gathered}
w_{\boldsymbol{u}}=\boldsymbol{N}_{\boldsymbol{u}} w_{\boldsymbol{u}} \quad \text { and } \boldsymbol{\nabla} w_{\boldsymbol{u}}=\boldsymbol{B}_{\boldsymbol{u}} w_{\boldsymbol{u}} \\
9 \\
\text { http://mc.manuscriptcentral.com/engcom }
\end{gathered}
$$


where, $\boldsymbol{N}_{\boldsymbol{u}}$ and $\boldsymbol{B}_{\boldsymbol{u}}$ are the basis function and derivative of the basis function associated with displacement, respectively. Substituting Eq.(34) and Eq.(35) into Eq.(23), the final discretized form of integral equations are obtained as

$$
\begin{gathered}
\int_{\Omega^{e}} \boldsymbol{B}_{\boldsymbol{u}}^{T} \boldsymbol{\sigma} d \Omega^{e}=\int_{\Omega^{e}} \boldsymbol{N}_{\boldsymbol{u}}^{T} \boldsymbol{f}_{\boldsymbol{b}} d \Omega^{e}+\int_{T_{t}^{e}} \boldsymbol{N}_{\boldsymbol{u}}^{T} \boldsymbol{t} d T_{\boldsymbol{f}_{\mathrm{ext}}^{u}}^{e} . \\
\boldsymbol{f}_{\text {int }}^{\boldsymbol{u}}=\boldsymbol{f}_{\text {ext }}^{\boldsymbol{u}}
\end{gathered}
$$

Let $\boldsymbol{N}_{\bar{e}}$ and $\boldsymbol{B}_{\bar{e}}$ are the basis function and derivative of the basis function associated with nonlocal equivalent strain $\bar{e}$, respectively. The final form of the Eq.(24) in terms of forces can be written as

$$
\begin{aligned}
& \bar{e}=\boldsymbol{N}_{\bar{e}} \bar{e}, \quad \boldsymbol{\nabla} \bar{e}=\boldsymbol{B}_{\bar{e}} \bar{e} \text { and } \nabla^{2} \bar{e}=\boldsymbol{G}_{\bar{e}} \bar{e} \\
& w_{\bar{e}}=\boldsymbol{N}_{\bar{e}} w_{\bar{e}}, \quad \nabla w_{\bar{e}}=\boldsymbol{B}_{\bar{e}} w_{\bar{e}} \quad \text { and } \boldsymbol{\nabla} w_{\bar{e}}=\boldsymbol{G}_{\bar{e}} w_{\bar{e}} \\
& \int_{\Omega^{e}} \boldsymbol{N}_{\bar{e}}^{T} \boldsymbol{N}_{\bar{e}} \bar{e} d \Omega^{e}+\int_{\Omega^{e}} a_{1} \boldsymbol{B}_{\bar{e}}^{T} \boldsymbol{B}_{\bar{e}} \bar{e} d \Omega^{e}-\int_{\Omega^{e}} a_{2} \boldsymbol{G}_{\bar{e}}^{T} d \boldsymbol{G}_{\bar{e}} \bar{e} d \Omega^{e}-\int_{\Omega^{e}} e \boldsymbol{N}_{\bar{e}}^{T} d \Omega^{e}=0 \\
& \boldsymbol{f}_{\text {int }}^{\bar{e}}=\boldsymbol{f}_{\text {ext }}^{\bar{e}}
\end{aligned}
$$

Initial and final values of $\kappa$ can be derived from experimental study.

$$
\begin{gathered}
\left\{\frac{\partial D}{\partial \kappa}\right\}_{i-1}= \begin{cases}0 & \text { iff } \bar{e}_{i_{1}} \leq \kappa_{0}, \\
\left\{\frac{\partial D}{\partial \kappa}\right\}_{i-1} & \text { iff } \bar{e}_{i-1}>\kappa_{0}\end{cases} \\
\left\{\frac{\partial \kappa}{\partial \bar{e}}\right\}_{i-1}= \begin{cases}1 & \text { iff } \bar{e}_{i_{1}} \leq \kappa_{0} \\
0 & \text { iff } \bar{e}_{i-1}>\kappa_{0}\end{cases}
\end{gathered}
$$

By estimating the value of yield function, we can easily calculate the constitutive matrix

$$
\tilde{\boldsymbol{E}}=\left\{\begin{array}{lr}
\boldsymbol{E} & \text { iff } F(\boldsymbol{\sigma}, \boldsymbol{q})<0, \\
\boldsymbol{E}_{\mathrm{ep}} & \text { iff } F(\boldsymbol{\sigma}, \boldsymbol{q})=0
\end{array}\right.
$$

Then, the linearization of primary and secondary variables at $i^{\text {th }}$ step with respect to previous step $i-1$ is given by

$$
\begin{gathered}
\boldsymbol{u}_{i}=\boldsymbol{u}_{i-1}+\delta \boldsymbol{u}_{i} \\
e_{i}=e_{i-1}+\left\{\frac{\partial e}{\partial \boldsymbol{\varepsilon}}\right\}_{i-1}^{T} \boldsymbol{B}_{\boldsymbol{u}} \delta \boldsymbol{u}_{i} \\
\boldsymbol{\varepsilon}_{i}=\boldsymbol{\varepsilon}_{i-1}+\boldsymbol{B}_{\boldsymbol{u}} \delta \boldsymbol{u}_{i} \\
\boldsymbol{\sigma}_{i}=\boldsymbol{\sigma}_{i-1}+\delta \boldsymbol{\sigma}_{i}
\end{gathered}
$$

where

$$
\delta \boldsymbol{\sigma}_{i}=\left[1-D_{i-1}\right] \tilde{\boldsymbol{E}}: \boldsymbol{B}_{\boldsymbol{u}} \delta \boldsymbol{u}_{i}-\left\{\frac{\partial D}{\partial \kappa}\right\}_{i-1}\left\{\frac{\partial \kappa}{\partial \bar{e}}\right\}_{i-1} \boldsymbol{N}_{\bar{e}} \delta \overline{\boldsymbol{e}}_{i} \tilde{\boldsymbol{E}}: \boldsymbol{\varepsilon}_{i-1}
$$


To represent the post peak softening behavior, we adopt the arc-length or the modified Riks method. This method is found to be more suitable to solve system of highly nonlinear equations and the stiffness matrix terms are all not positive definite. The following steps have been considered for solving the nonlinear equations. At the first, the step and increment values are initialized. The incremental displacement is set as $\boldsymbol{u}=0$. The load incremental factor $\gamma$ and initial applied load $P_{0}$ are user defined. The applied load is then calculated as $\gamma P_{0}$. For subsequent steps, $\gamma, \boldsymbol{u}$, and $\bar{e}$ are updated by estimating the values of $\Delta \gamma, \Delta \boldsymbol{u}$, and $\Delta \bar{e}$ respectively. As and when, the force residual, $\boldsymbol{R}$ occurs to be greater than given tolerance, crisfield arc-length approach is invoked. The value of incremental load multiplier $\Delta \gamma$ is computed using $\Delta \gamma_{i}=\frac{(d S)^{2}-\left[\boldsymbol{u}_{i}-\boldsymbol{u}_{i-1}\right]^{\prime}\left[\Delta \boldsymbol{u}_{i-1}+d \boldsymbol{u}_{\boldsymbol{R}}\right]}{\left[\boldsymbol{u}_{i}-\boldsymbol{u}_{i-1}\right]^{\prime} d \boldsymbol{u}_{P}}$, where $d S$ is computed as $d S=$ $\Delta \gamma_{i-1} \sqrt{d \boldsymbol{u}_{P}^{\prime} d \boldsymbol{u}_{P}}$. The incremental values $d \boldsymbol{u}_{\boldsymbol{R}}$ and $d \boldsymbol{u}_{P}$ are given as $d \boldsymbol{u}_{\boldsymbol{R}}=[\boldsymbol{K}]^{-1}[\boldsymbol{R}]$, $d \boldsymbol{u}_{P}=[\boldsymbol{K}]^{-1}[P]$. With the help of $\Delta \gamma_{i}$, updated $\Delta \boldsymbol{u}_{i}$ is calculated as $\Delta \boldsymbol{u}_{i}=d \boldsymbol{u}_{\boldsymbol{R}}+\Delta \gamma_{i} d \boldsymbol{u}_{P}$ and the updated displacement and load are given by $\boldsymbol{u}_{i}=\boldsymbol{u}_{i-1}+\Delta \boldsymbol{u}_{i}, P=\gamma_{i} * P_{0}$, where $\gamma_{i}$ is calculated as $\gamma_{i}=\gamma_{i-1}+\Delta \gamma_{i}$. The updated tangent stiffness matrix $[\boldsymbol{K}]$ and Residual $[\boldsymbol{R}]$ are then computed. The normx of the residual $[\boldsymbol{R}]$ is then calculated. If this value if found to be less than the given tolerance value, the displacement $\boldsymbol{u}_{i}$ is considered to be converged. If not then the above steps are repeated till the convergence is reached. The same steps have been applied to calculate $\bar{e}$. 


\section{$6 \quad$ Numerical results and discussions}

\subsection{A bar under uniaxial tension with a linear damage}

An axially deforming bar of length $L=100 \mathrm{~mm}$ fixed at one end and subjected to an uniaxial pure tensile load is considered for the analysis. The cross-sectional area $A$ has been reduced by a factor of 0.1 between $x=-\frac{l}{2}$ and $x=+\frac{l}{2}$. The bar has uniform material properties along the length and these are taken as Elastic modulus $E=20 G P a$, plastic modulus $H=0.2 G P a$, $\kappa_{0}=1 \times 10^{-4}, \kappa_{c}=12 \times 10^{-4}$, and characteristics length $l=1 \mathrm{~mm}$. Two cases are considered for the analysis, first a linear softening model and seconf an exponential softening model.

\subsection{Case A: Linear damage}

A linear damage evolution law is given by

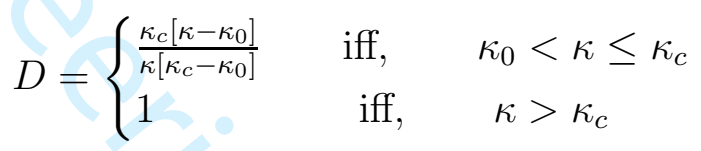

An Isogeometric FE analysis is performed for the bar. Fig 2 shows the variation of damage with respect to the length of the bar. Here, the damage is accounting for the deterioration in material properties, equivalent of some physical mechanisms such as microcracking in the material at different stages of loading. At every increment of the load step, the probability of failure is increasing, and if it is observed closely, the width of damage zone in Fig [2] has been increased by a significant amount.

In Figs 3 and 4 , plastic and total strains measured have been plotted along the length of the bar for 198 elements. These figures are plotted to compare the amount of plastic strain accumulated in the body with respect to the total strain. Generally, quasi-brittle materials show very less inclination towards plastic behavior. However, in this example, the amount of plastic strain accumulated is more than the elastic strain. Furthermore, the peak value of the strain in a reduced area delineates about the coalescing of a micro-crack to a macrocrack, whereas in other sections, there is no evidence of coalescing. Finally, a propagation of macro-crack is leading to the failure of the material. 


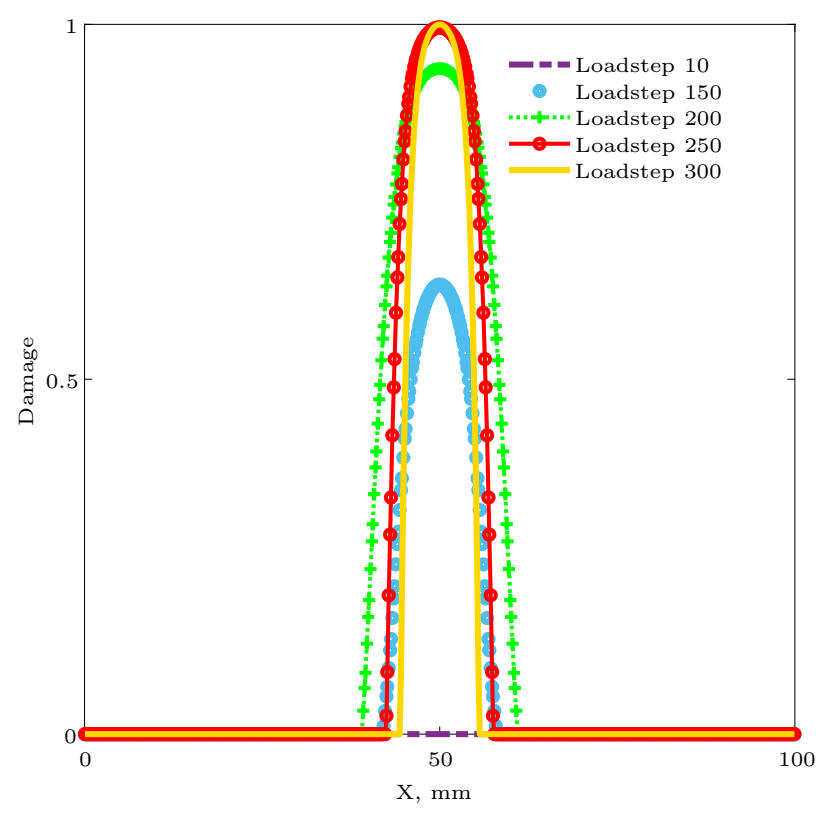

Figure 2: Damage curve at various load steps in axial bar with a central notch subjected to tensile loading

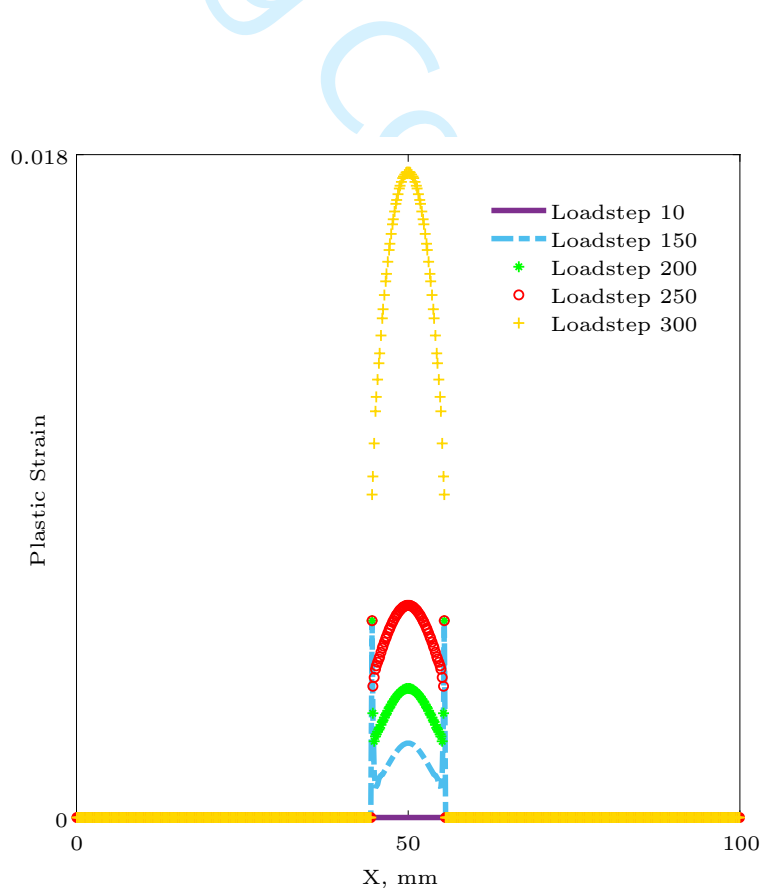

Figure 3: Plastic strain curve at various load steps for axial bar under tensile loading 


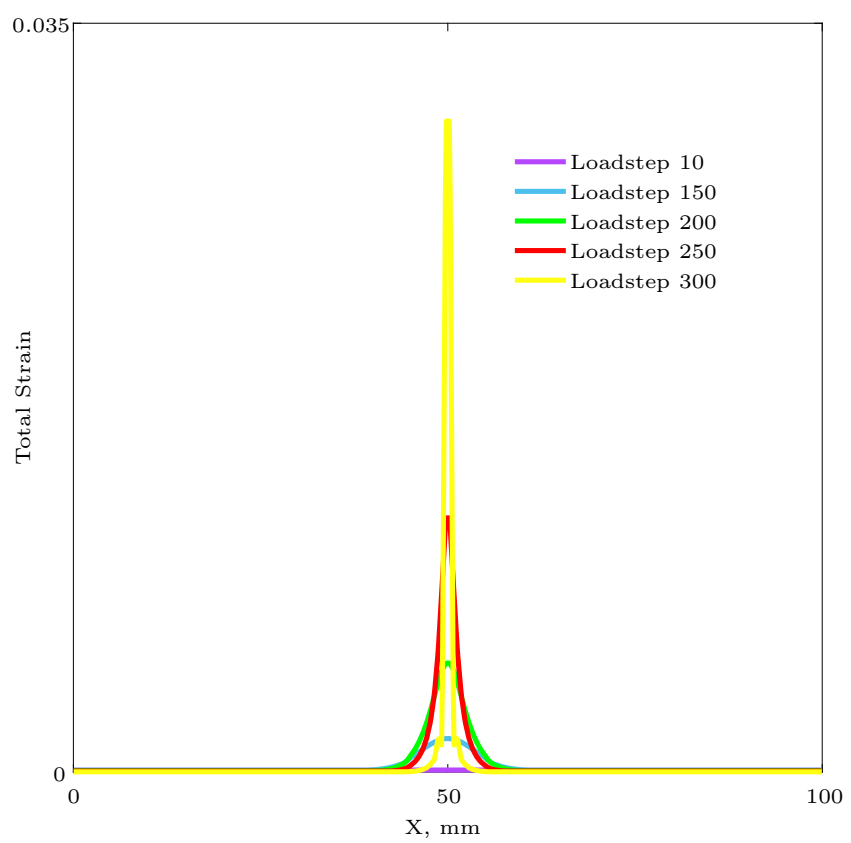

Figure 4: Total strain curve at various load steps

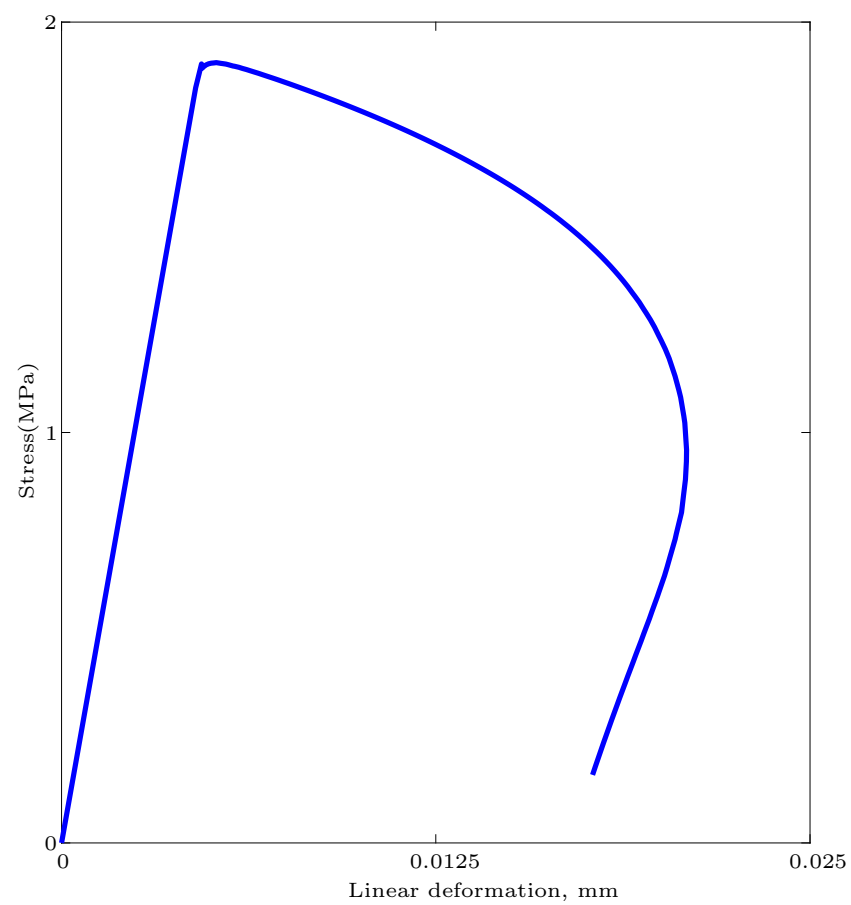

Figure 5: Stress-linear deformation curve for the axial bar under tensile loading 


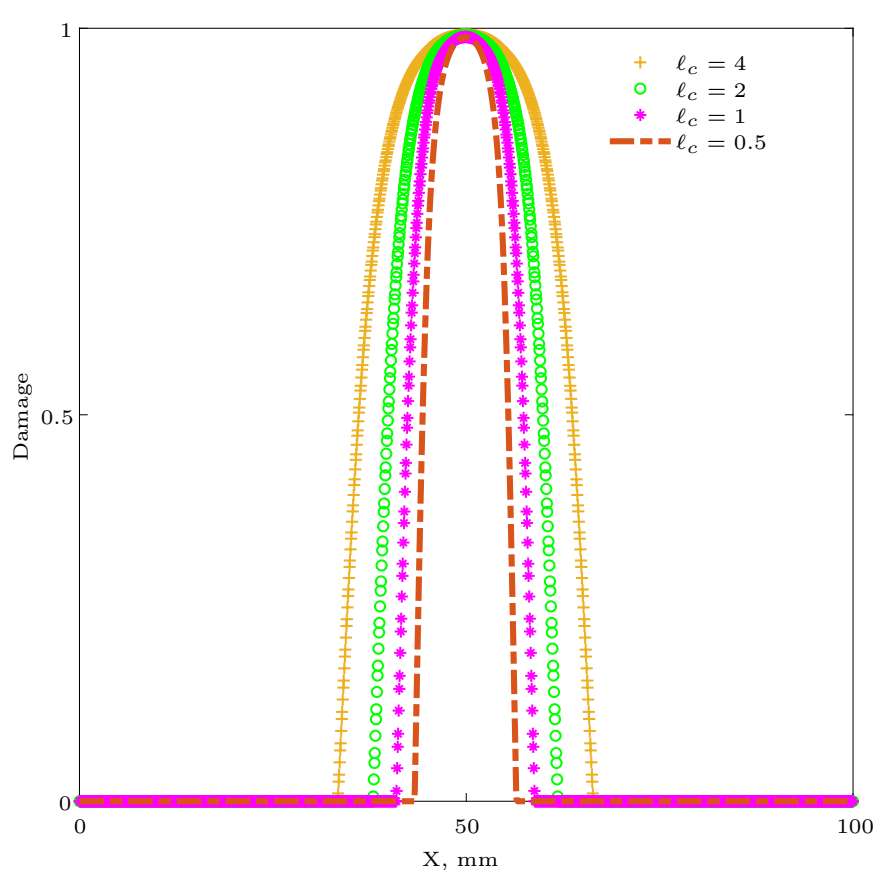

Figure 6: Damage curve at various characteristics length scales

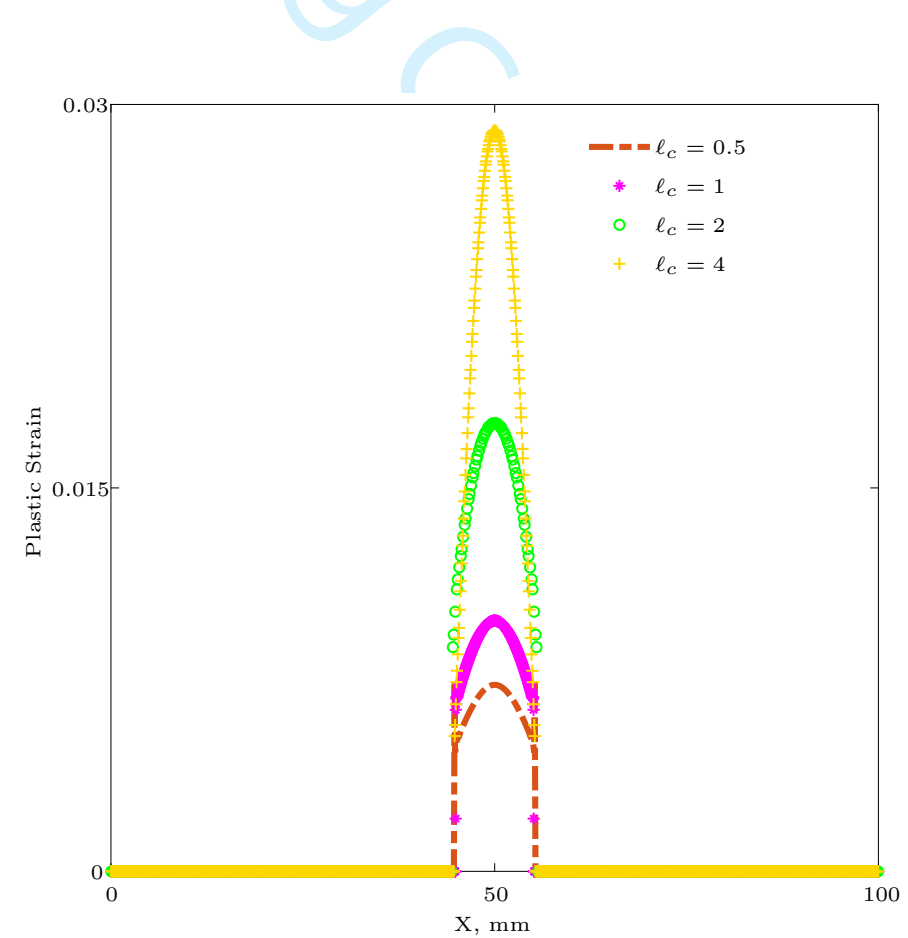

Figure 7: Plastic strain curve at various characteristics length scales 


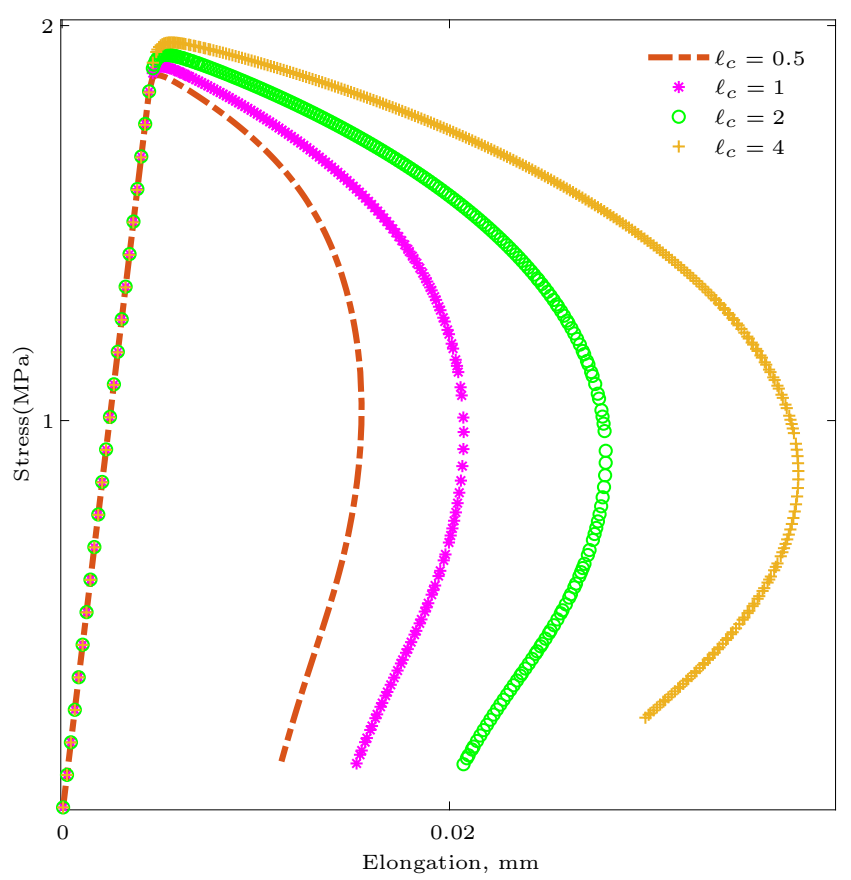

Figure 8: Stress-linear deformation curve at various characteristics length scales

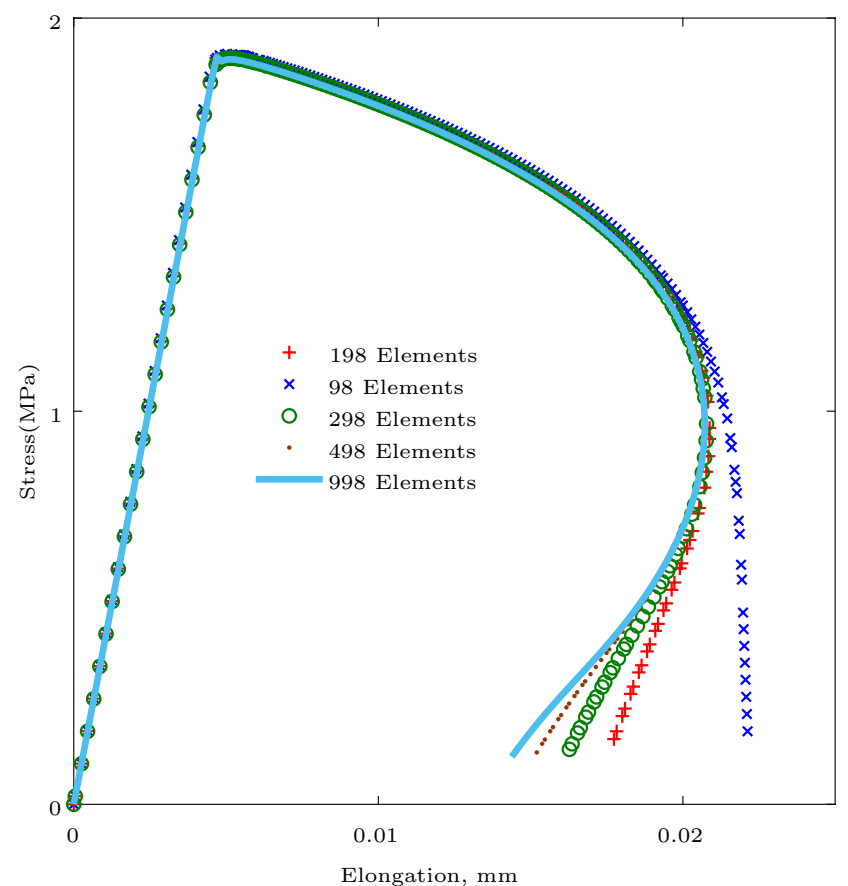

Figure 9: Stress-linear deformation curve at various elements 
The stress-linear deformation curve in Fig $[$ is presented to demonstrate that the damage and plasticity are causing softening behavior in the body. The following graphs compare the behavior of the body concerning the variation in characteristics length scales for 498 elements. The damage evolution (Fig (6), plastic strain evolution (Fig,7), and the load-elongation curve (Fig 8) are obtained by considering various characteristics lengths for 498 elements. It concludes that an increased characteristic length in material leads to a higher deformation since the micro deformation finds number of ways to grow over a large area. Fig 9 summaries that, while unloading, a higher number of elements accumulated less amount of inelastic strain. Here, the characteristic length scale is kept constant at $1 \mathrm{~mm}^{2}$ to demonstrate the effects of the number of elements. In all these cases the trends in distribution of damage and strain with length scale parameter and with iterations are similar to those presented in litreature. In linear elastic cases of present model the results were matching with those in litreature [41].

\subsection{Case B: Exponential damage law}

An exponential damage evolution law is given by

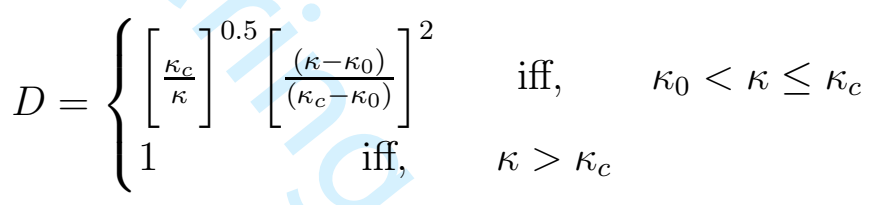

For the same bar example presented above, an exponential law in damage is considered for analysis to compare the accumulated strain, stress, and damage zone. Figs 10 to 13 represent the behavior of a body consisting of 198 elements with $l_{c}=1 \mathrm{~mm}^{2}$. Fig 10 concludes that with an exponential damage law, a crack in the body starts propagating sharply instead of coalescing the micro-cracks.

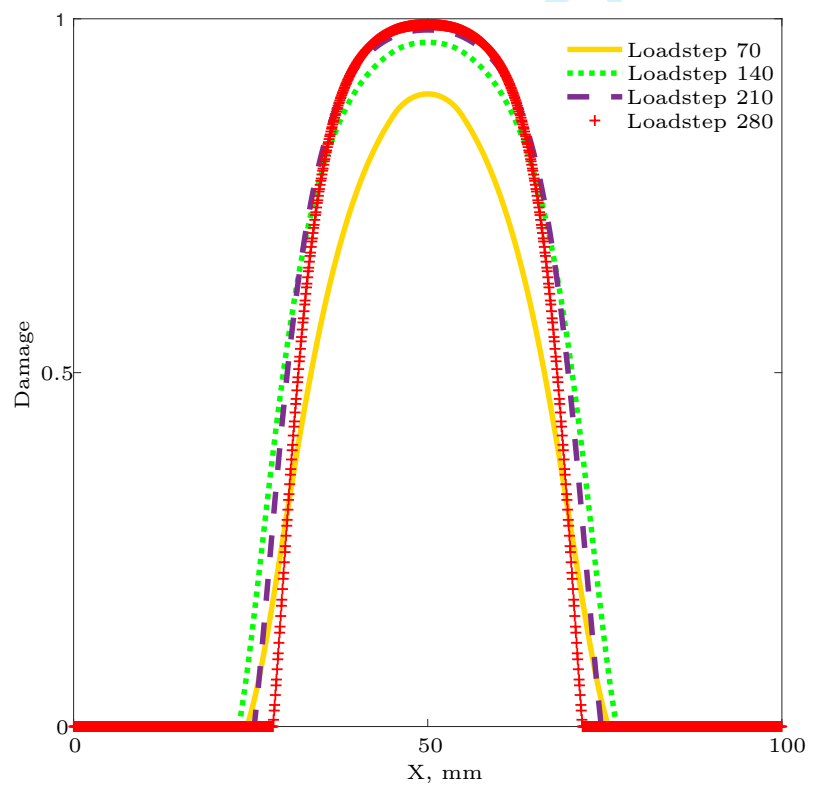

Figure 10: Damage curve at various load steps 


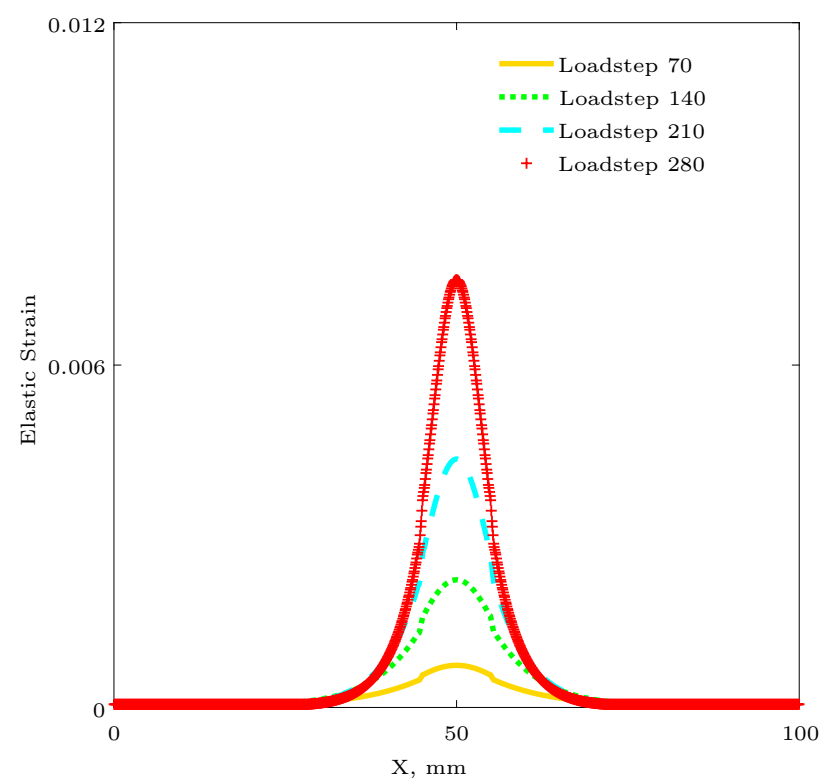

Figure 11: Evolution of elastic strain at various load steps

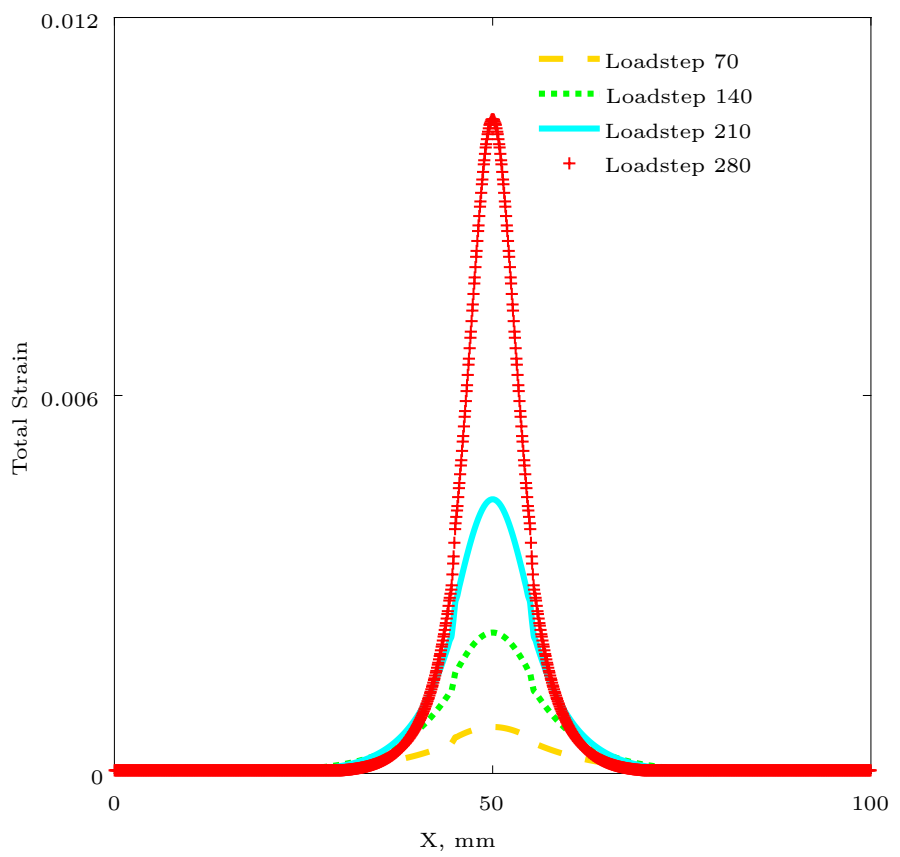

Figure 12: Total strain curve at various load steps 
2

3

4

5

6

7

8

9

10

11

12

13

14

15

16

17

18

19

20

21

22

23

24

25

26

27

28

29

30

31

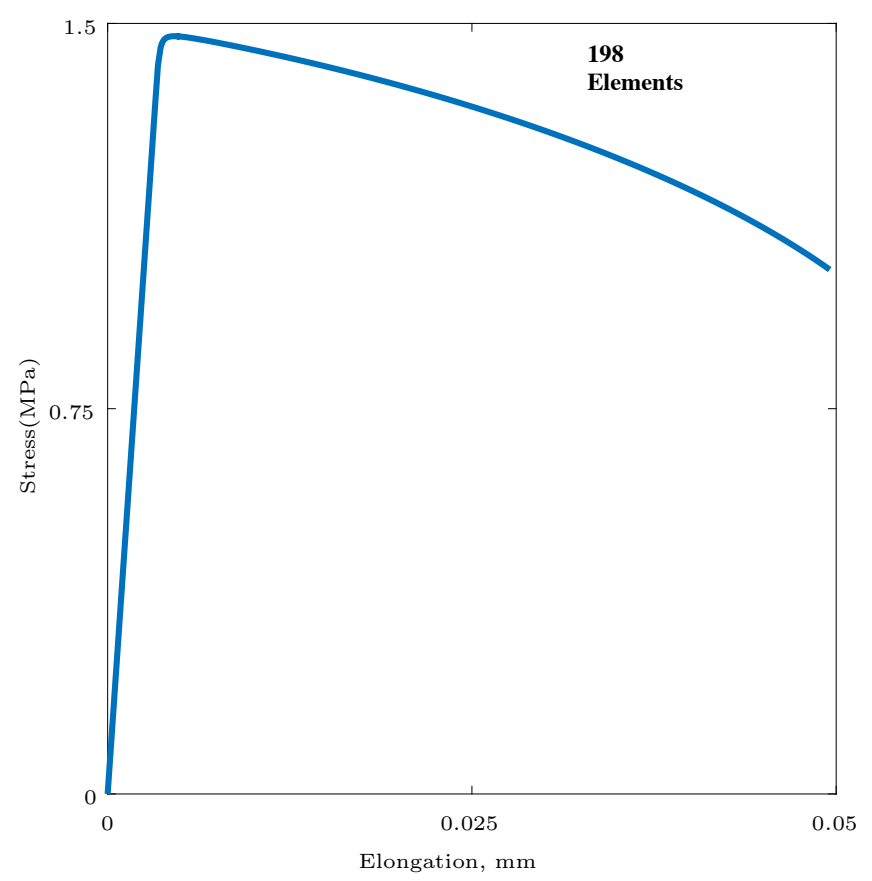

Figure 13: Stress-linear deformation curve

Fig 11] shows that the accumulated elastic strain is higher than the plastic strain. It concludes that the failure in a body is governed by the damage in the case of an exponential damage law. Figs 14 to 17 represent the behavior of a body with 498 elements. With an exponential damage law, Fig 14 concludes that with higher elements, crack in a body starts coalescing sharply instead of propagation of the macro-cracks. However, with a linear damage law, the same behavior is not observed, see Fig 6. In all these cases the trends in distribution of damage and strain with length scale parameter and with iterations are similar to those presented in litreature. In linear elastic cases of present model the results were matching with those in litreature [41]. 


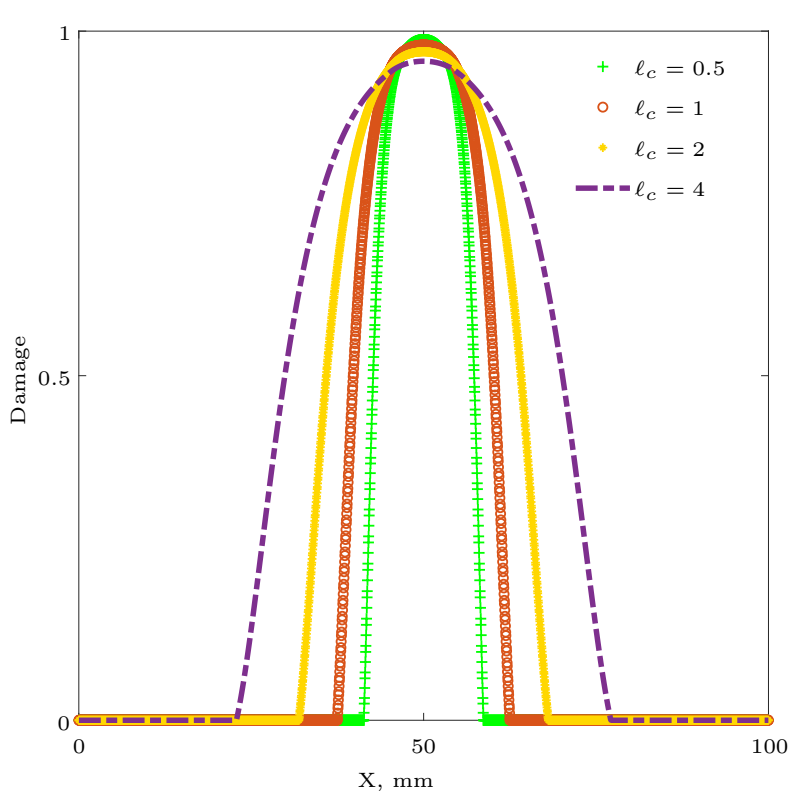

Figure 14: Damage curve at various characteristics length scales

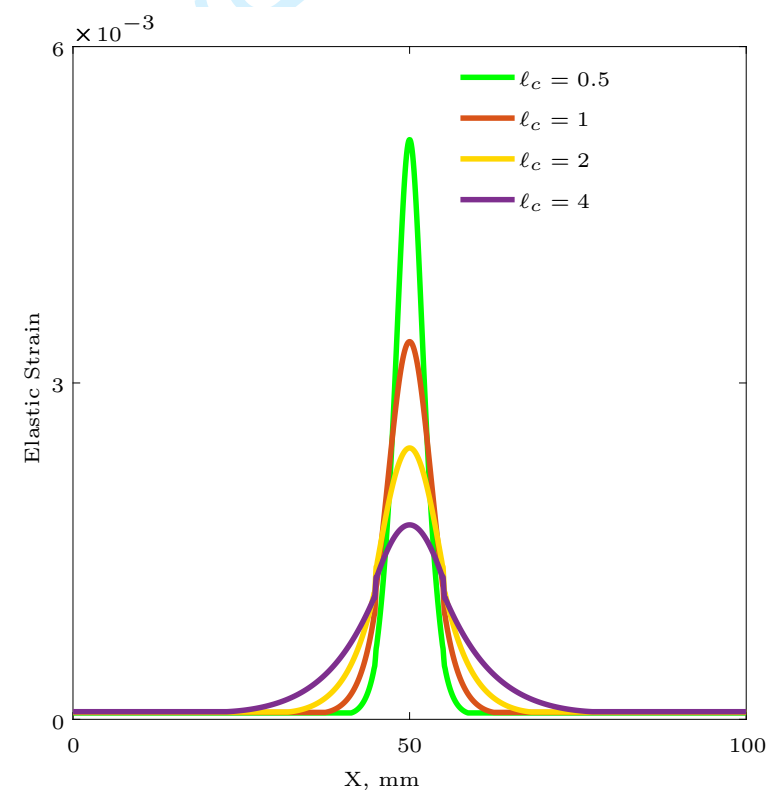

Figure 15: Elastic strain curve at various characteristics length scales

From Fig.15, it is observed that with an increase in the number of elements, accumulated plastic strain is higher which concludes that the failure is governed by plasticity. 


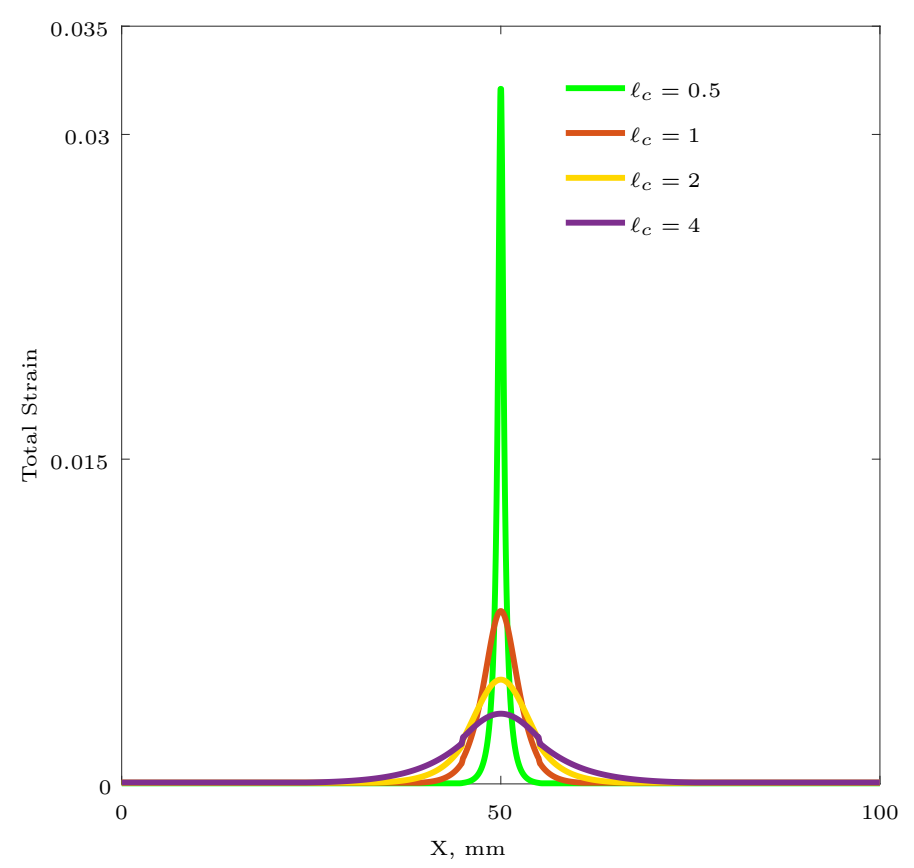

Figure 16: Total strain curve at various characteristics length scales

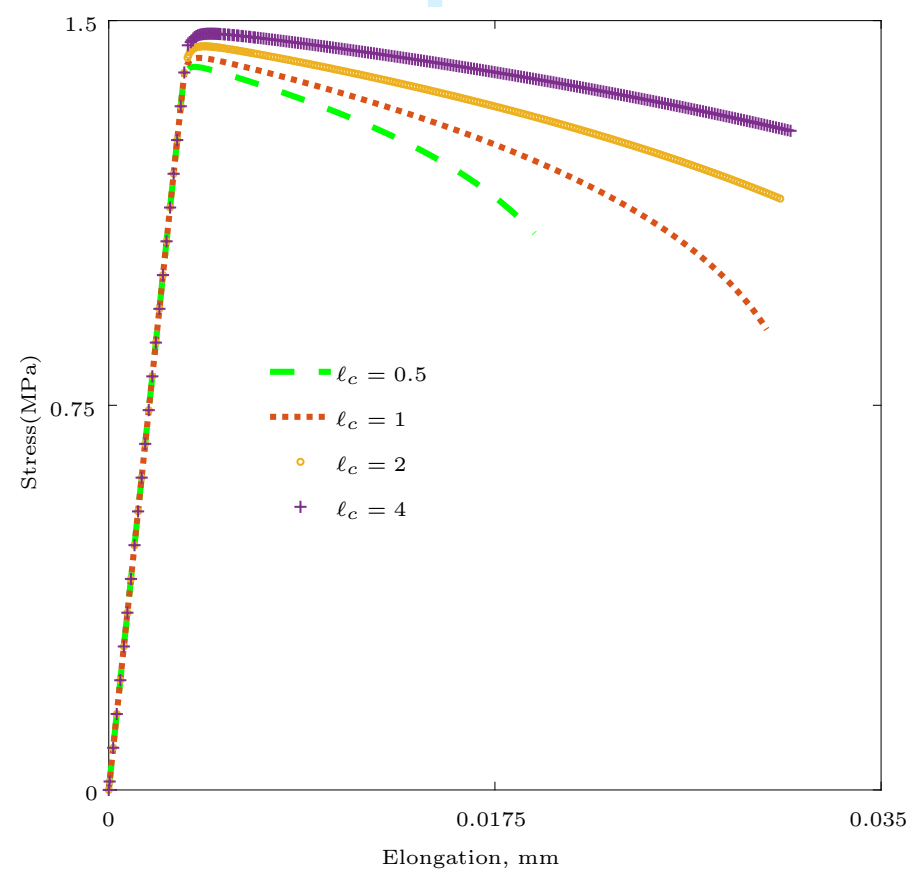

Figure 17: Stress-Linear deformation curve at various characteristic length scales 


\subsection{A plate with hole under tension}

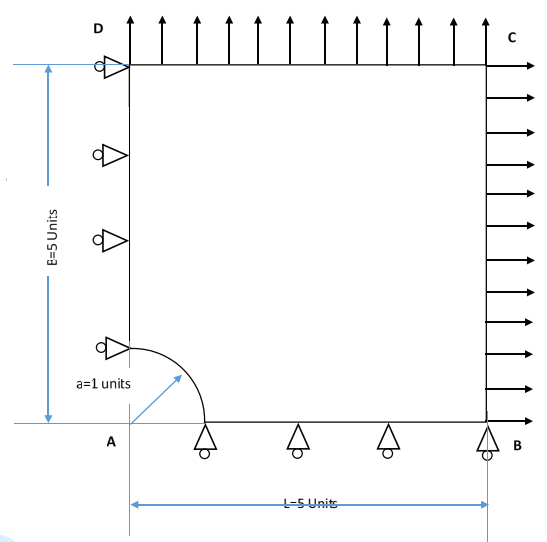

Figure 18: Geometry of the plate with circualr hole considered for the analysis

\begin{tabular}{ccccccc}
\hline$I$ & $P_{I, 1}$ & $P_{I, 2}$ & $P_{I, 3}$ & $w_{I, 1}$ & $w_{I, 2}$ & $w_{I, 3}$ \\
\hline 1 & $(0,1)$ & $(0,3)$ & $(0,5)$ & 1 & 1 & 1 \\
2 & $(\sqrt{2}-1,1)$ & $(0.75,3)$ & $(5,5)$ & $(1+1 / \sqrt{2}) / 2$ & 1 & 1 \\
3 & $(1, \sqrt{2}-1)$ & $(3,0.75)$ & $(5,5)$ & $(1+1 / \sqrt{2}) / 2$ & 1 & 1 \\
4 & $(1,0)$ & $(3,0)$ & $(5,0)$ & 1 & 1 & 1 \\
\hline
\end{tabular}

Table 1: Control points and weights for plate with circular hole

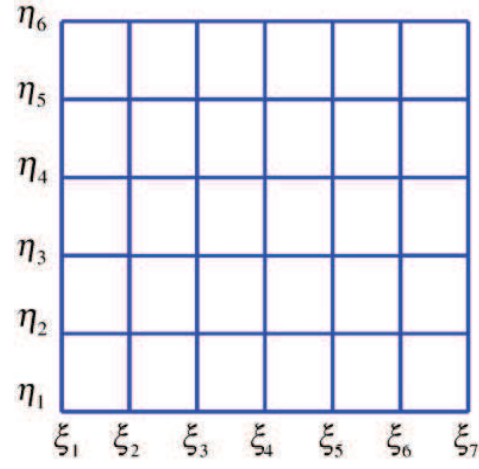

(a)

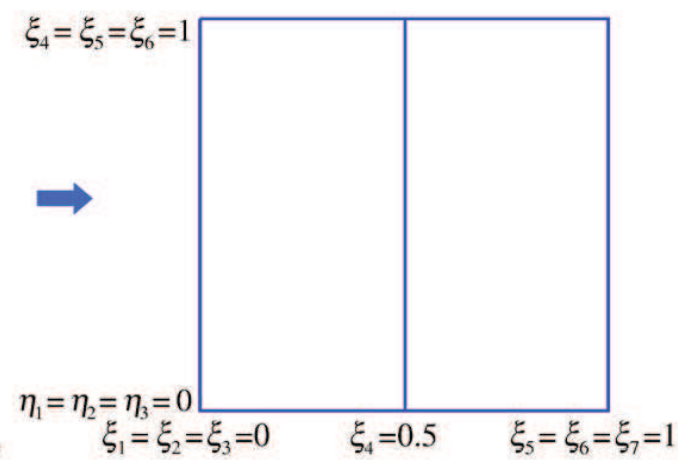

(b)

Figure 19: Knot vectors defined in (a) Index space and (b) Parameter space

A square plate with a central circular hole as shown in Fig. [18 is considered for the analysis. The plate is subjected to a uniform displacement of $0.2 \mathrm{~mm}$ at both edges up to $0.26 \mathrm{~mm}$. The material properties taken are youngs modulus $E=20 G P a$, Hardening modulus $h=0.2 G P a$, length scale $l=1 \mathrm{~mm}^{2}$, and Poisson's ratio $\nu=0.2$. The purpose of this example is to demonstrate the applicability of the method to two dimensional problems. Due to its symmetry, one fourth of the plate is modeled. A rational quadratic basis is used to 
describe NURBS described square plate with circular hole. Knot vectors $\xi \times \zeta$ of coarse mesh with two quadratic elements are defined as $\xi=\{0,00,0.5,1,1,1\}$ and $\zeta=\{0,0,0,1,1,1\}$ which define the index space and parameter space as shown in Fig.19.

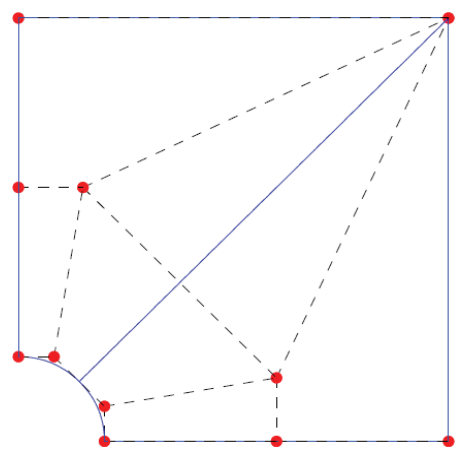

(a)

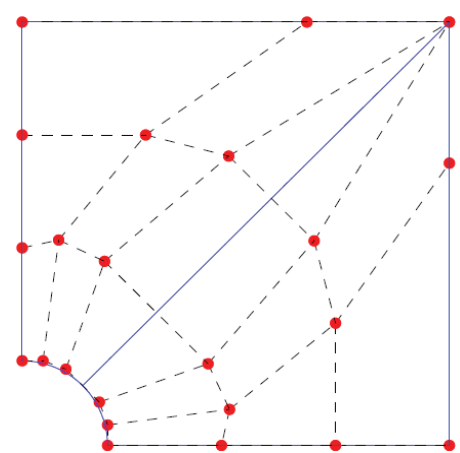

(b)

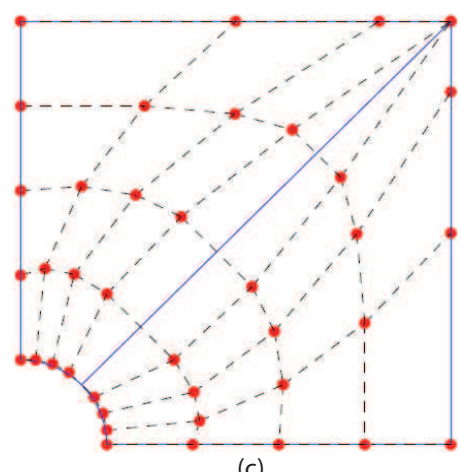

(c)

Figure 20: Coarse mesh and Control net for the plate with the hole example (a) Quadratic (b) Cubic (c) Quartic

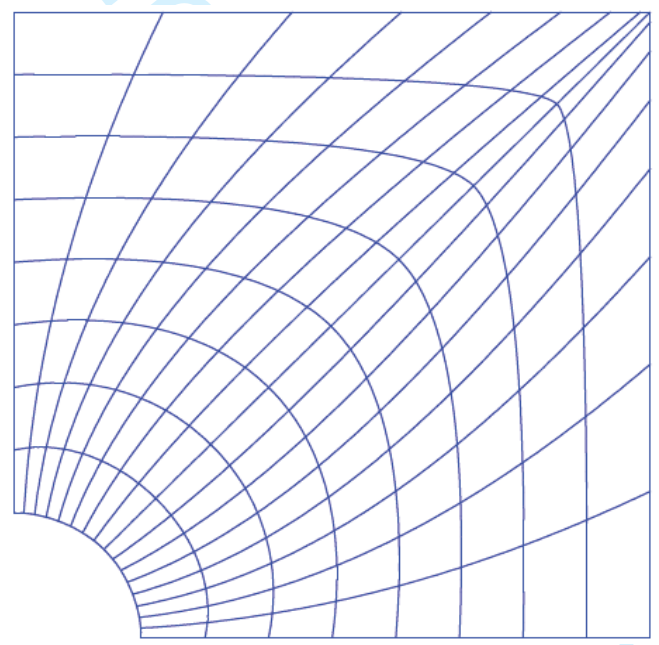

Figure 21: Meshing of the quarter plate. $16 \times 8$ elements are considered

The coarse mesh and possible control nets for this case are as shown in Fig 20. The control point locations and their respective weights are given in Table 1 . The control point connectivity and knot vector connectivity can be suitably defined based on choice of control points and non zero knot spans. Using this information the meshes are generated. The meshing of the domain is obtained using $16 \times 8$ elements as shown in Fig 21. Following the standard procedure of isogemetric analsyis, as discussed in earlier sections, the NURBS approximation in 2D and their mapping are obtained. Gauss quadratue rule of $3 \times 3$ is adopted for numerical integration. The geometry and approximation are accurately modeled with the same approximation. An exponential damage evolution law of the following form is considered.

$$
D=1-\frac{\kappa_{0}}{\kappa}\left[1-\alpha+\alpha e^{\beta\left(\kappa-\kappa_{0}\right)}\right]
$$


where, $\kappa_{0}=2.1 e-04, \alpha=0.96$ and $\beta=350$ are the damage parameters, and $e$ is the local equivalent strain,

$$
e=\frac{K-1}{2 K[1-2 \nu]} I_{1}+\frac{1}{2 K}\left\{\frac{[K-1]^{2}}{[1-2 \nu]^{2}} I_{1}^{2}-\frac{12 K}{[1+\nu]^{2}} J_{2}\right\}^{1 / 2}
$$

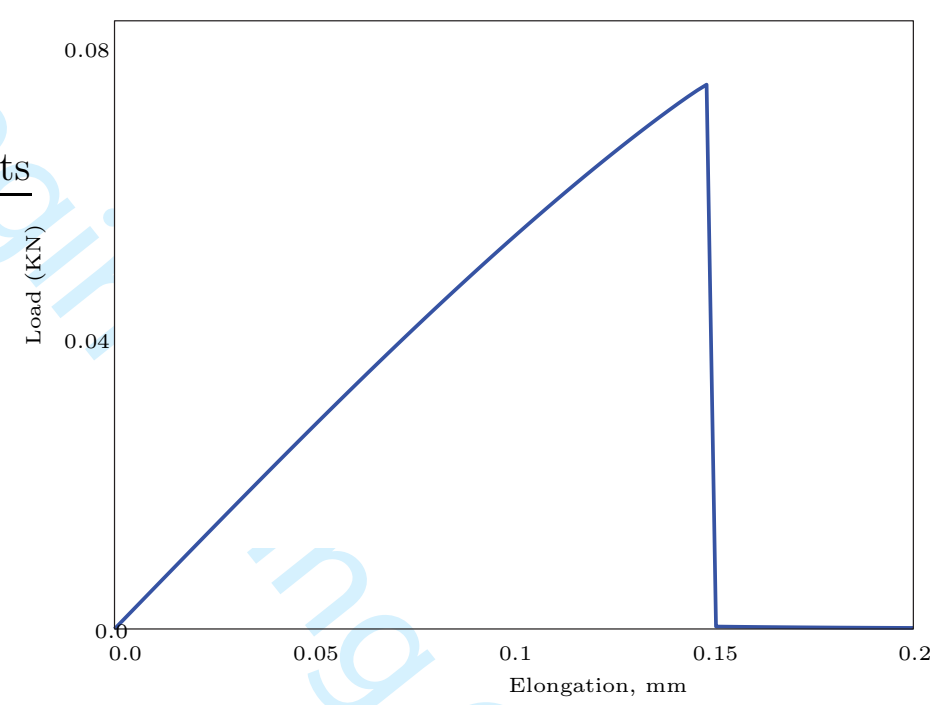

Figure 22: Load-elongation curve for the plate with central hole example
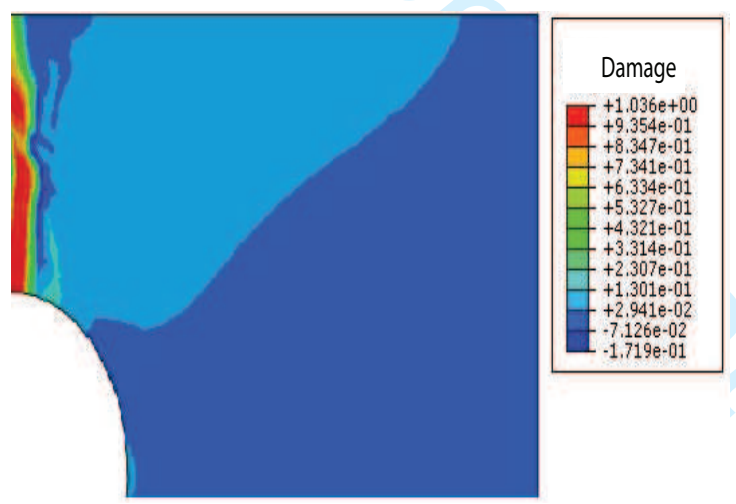

Figure 23: Distribution of damage for the plate with central hole example

where, $K$, i.e., the compression to tensile strength ratio, is taken as 10. An exponential damage law ensures damage value never reaches 1 ; hence, this implies that material will not be completely fractured. The local equivalent strain is evaluated using Von-Mises equation. A plot of load elongation curve and damage is shown in Fig 22 and Fig 23. It is evident that damage in the plate is high since quasi-brittle materials dissipate the energy through damage process. 


\section{Conclusion}

In this paper, we have presented a nonlocal gradient plasticity damage model to predict a crack pattern in quasi-brittle materials. To validate the utility of model, a few examples have been solved. This model account for the elastic and inelastic deformation, however, it limits to provide a relation between temperature rise and energy dissipation. To capture the post-peak behavior of the material, we have implemented an Arc-length method. With the solved examples in one and two dimensions, it can be concluded that the present model allows a successful description of several aspects of the nonlocal elastoplastic behavior of the quasi-brittle materials. It also permits a successful evaluation of the size effects based on the simulations conducted by varying the number of elements in the system.

\section{References}

[1] L. M. Kachanov. On rupture time under condition of creep. Izvestia Akademi Nauk SSSR, Otd. Tekhn. Nauk, 8:26-31, 1958.

[2] Y. N. Rabotnov. Creep problems in structural members. North-Holland, Amsterdam, 1969.

[3] D. Krajcinovic. Damage mechanics. Mechanics of Materials, 8:117-197, 1989.

[4] Z. P. Bazant, T. Belytschko, and T. P. Chang. Continuum theory for strain softening. Journal of Engineering Mechanics, 110:59-64, 1984.

[5] G. Pijaudier-Cabot and Z. P. Bazant. Nonlocal damage theory. Journal of Engineering Mechanics, 113:1512-1533, 1987.

[6] Z.P. Bazant. Why continuum damage is nonlocal: Micromechanics arguments. Journal of Engineering Mechanics, 117:76-95, 1991.

[7] A.C. Eringen. On differential equations of nonlocal elasticity and solutions of screw dislocation and surface waves. Journal of Applied Physics, 54:4703-4710, 1983.

[8] A.C. Eringen. Microcontinuum field theories-1. Foundations and solids. Springer- Verlag, Newyork, 1998.

[9] M. Jirasek. Nonlocal theories in continuum mechanics. Acta Polytechnica, 44(5-6):16-34, 2004.

[10] J.N. Reddy. Nonlocal theories for bending, buckling and vibration of beams. International Journal of Engineering Science, 45:288-307, 2007.

[11] O. Civalek and C. Demir. Bending analysis of microtubules using nonlocal EulerBernoulli beam theory. Applied Mathematical Modelling, 35:2053-2067, 2011.

[12] H. Askes, A. Suiker, and L. Sluys. A classification of higher-order strain-gradient modelslinear analysis. Archive of Applied Mechanics, 72:171-188, 2002. 
[13] N. Kirchner and P. Steinmann. Mechanics of extended continua: modeling and simulation of elastic microstretch materials. Computational Mechanics, 40(4):651-666, 2006.

[14] C. Giry, F. Dufour, and J. Mazars. Stress-based nonlocal damage model. International Journal of Solids and Structures, 48:3431-3443, 2011.

[15] E.Amanatdou and A. Aravas. On microstructural origin of certain inelastic models. Journal of Engineering Material technology, 106:326-330, 1984.

[16] M.G.D. Geers, V. Kouznetsova, and W.A.M Brekelmans. Gradient enhanced computational homogenization for the micro macro scale transition. Journal of Physics, 11(4):145-152, 2001.

[17] V.G. Kouznetsova. Computational homogenization for the multiscale analysis of multipahse materials. PhD, thesis, Technische Universiteit, Eindhoven, 2002.

[18] V.G. Kouznetsova, M.G.D. Geers, and W.A.M. Brekelmans. Multiscale second order computational homogenization of multiphase materials: A nested finite element solution strategy. Computer Methods in Applied Mechanics and Engineering, 193:5525-5550, 2004.

[19] C. Polizzotto. Gradient elasticity and non standard boundary conditions. International Journal for Solids and Structures, 40:7399-7423, 2003.

[20] N. A. Fleck and J.W. Hutchinson. Strain gradient plasticity. Adv. app.Mech, 33, 1996.

[21] I. Aranson, V. Kalatsky, and V. Vinokur. Continuum field description of crack propogation. Physical Review Letters, 85:118-121, 2000.

[22] A. Karma, D. A. Kessler, and H. Levine. Phase-field model of mode iii dynamic fracture. Physical Review Letters, 87:45501, 2001.

[23] C. Miehe, M. Holfacker, and F. Welschinger. A phase field model for rate-independent crack propogation: Robust algorithmic implementation based on operator splits. Computer Methods in Applied Mechanics and Engineering, 199:2765-2778, 2010a.

[24] N. Mozaffari and G. Z. Voyiadjis. Nonlocal damage model using phase-field method: Theory and applications. International Journal of Solids and Structures, 50:3136-3151, 2013.

[25] E. Sarvaramini, M.B. Dusseault, M. Komijani, and R. Gracie. A nonlocal plasticity model of stimulated volume evolution during hydraulic fracturing. International Journal of Solids and Structures, 159:111-125, 2019.

[26] M. Kenawy, S. Kunnath, S. Kolwankar, and A. Kanvinde. Concrete uniaxial nonlocal damage plasticity model for simulating post peak response of rcc beam column under cyclic loading. Journal of Structural Engineering, 146:1-21, 2020. 
[27] M. Jirasek and R. Desmorat. Localization analysis of nonlocal models with damage dependent nonlocal interaction. International Journal of Solids and Structures, 146:1$21,2020$.

[28] M. Kurumatania, Y. Soma, and K. Terada. Simulations of cohesive fracture behavior of reinforced concrete by a fracture mechanics based damage model. Engineering Fracture Mechanics, 206:392-407, 2019.

[29] L.H. Poh and S. Swaddiwudhipong. Over-nonlocal gradient enhanced plastic-damage model for concrete. International Journal of Solids and Structures, 46:4369-4378, 2009.

[30] P. Grassl and M. Jirásek. Damage-plastic model for concrete failure. International Journal of Solids and Structures, 43:7166-7196, 2006a.

[31] S.M. Vrech and G. Etse. Gradient and fracture energy-based plasticity theory for quasibrittle materials like concrete. Computer Methods in Applied Mechanics and Engineering, 199:136-147, 2009.

[32] A.S. Chiarelli, J.F. Shao, and N. Hoteit. Modeling of elasto plastic damage behavior of a clay stone. International Journal of Plasticity, 19:23-45, 2003.

[33] G.D. Nguyen, J. Belnoue, and A. M. Korsunsky. Coupled damage plasticity modelling of ductile failure in an aluminium alloy. Applied Mechanics and Materials, 784:266-273, 2015.

[34] G. Zhang and K. Khandelwal. Modeling of nonlocal damage plasticity in beams using isogeometric analysis. Computers and Structures, 165:76-95, 2016.

[35] T.J.R. Hughes, J.A. Cottrell, and Y. Bazilevs. Isogeometric analysis: Cad, finite elements, nurbs, exact geometry and mesh refinement. Comput. Methods Appl. Mech. Engg., 194:4135-4195, 2005.

[36] M.J. Borden, T.J.R. Hughes, C.M. Landis, and C.V. Verhoosel. A higher-order phasefield model for brittle fracture: Formulation and analysis within the isogeometric analysis framework. Comput. Methods Appl. Mech. Engg., 273:100-118, 2014.

[37] A. Sarikaya and R.E. Erkmen. A plastic-damage model for concrete under compression. International Journal of Mechanical Sciences, 150:584-593, 2019.

[38] L.J. Sluys and $\mathrm{R}$ de Borst. Dispersive properties of gradient-dependent and ratedependent media. 1994;. Mechanics of Materials, 18(2):131-149, 1994.

[39] M. Jirásek, S. Rolshoven, and P. Grassl. Size effect on fracture energy induced by nonlocality. International Journal for Numerical and Analytical Methods in Geomechanics, 28(7-8):653-670, 2004.

[40] A. Simone, H. Askes, and L.J. Sluys. Incorrect initiation and propagation of failure in non-local and gradient-enhanced media. International Journal of Solids and Structures, 41:351-363, 2004. 
[41] B. Umesh and A. Rajagopal. Higher order continuous approximation for the assessment of nonlocal-gradient based damage model. Mechanics of advanced materials and structures, 26:1671-1682, 2019.

[42] J.Y. Wu, J. Li, and R. Faria. An energy release rate-based plastic-damage model for concrete. International Journal of Solids and Structures, 43:583-612, 2006.

[43] L. Jason, A. Huerta, G.P. Cabota, and S. Ghavamian. An elastic plastic damage formulation for concrete: Application to elementary tests and comparison with an isotropic damage model. Computer Methods in Applied Mechanics and Engineering, 52:7077$7092,2006$.

[44] J. Lee and G.L. Fenves. A return-mapping algorithm for plastic-damage models: 3D and plane stress formulation. International Journal for Numerical Methods in Engineering, 50:487-506, 2001.

[45] J.C. Simo and T.J.R. Hughes. Computational inelasticity. Springer-Verlag New York, 7(1), 1998. 